\title{
Late Pleistocene to Holocene activity at Bakening volcano and surrounding monogenetic centers (Kamchatka): volcanic geology and geochemical evolution
}

\author{
F. Dorendorf ${ }^{\mathrm{a}}$, T. Churikova ${ }^{\mathrm{b}}$, A. Koloskov ${ }^{\mathrm{b}}, \mathrm{G}$. Wörner ${ }^{\mathrm{a}, *}$ \\ ${ }^{\mathrm{a}}$ Geochemisches Institut, University of Göttingen, Goldschmidt Strasse 1, 37077 Göttingen, Germany \\ ${ }^{\mathrm{b}}$ Institute of Volcanic Geology and Geochemistry FED RAN, Piip Avenue 9, Petropavlovsk-Kamchatsky, Russia
}

Received 4 August 1999; accepted 10 April 2000

\begin{abstract}
The different roles of variable mantle sources and intra-crustal differentiation processes at Bakening volcano (Kamchatka) and contemporaneous basaltic monogenetic centers are studied using major and trace elements and isotopic data.

Three suites of volcanic activity are recognized: (1) plateau basalts of Lower Pleistocene age; (2) andesites and dacites of the Bakening volcano, the New Bakening volcano dacitic centers nearby; and (3) contemporaneous basaltic cinder cones erupted along subduction zone-parallel N-S faults. Age-data show that the last eruptions in the Bakening area occurred only 6001200 years ago, suggesting the volcano is potentially active.

Major element variations and petrographic observations provides evidence for a fractionation assemblage of olivine, clinopyroxene, \pm plagioclase, \pm magnetite (?) within the basaltic suite. The fractionation in the andesites and dacites is dominated by amphibole, clinopyroxene, orthopyroxene and plagioclase plus minor amounts of magnetite and apatite. The youngest cpx-opx-andesites of Bakening main volcano deviate from that trend. Their source was probably formed by mixing of basaltic magmas into the silicic magma chamber of the Bakening volcano. Overall trace element patterns as well as the $\mathrm{Sr}-\mathrm{Nd}-$ $\mathrm{Pb}$ isotopic compositions are quite similar in all rocks despite large differences in their chemical composition (from basalt to rhyodacite). In detail however, the andesite-dacites of the central Bakening volcano show a stronger enrichment in the more incompatible elements and depletion in HREE compared to the monogenetic basaltic centers. This results in a crossing of the REE-pattern for the two suites. The decrease in the HREEs can be explained by amphibole fractionation. A slab component is less likely because it would result in fractionation of the HREE from each other, which is not observed. The higher relative amounts of LILE in the dacitic and the large scatter in the basaltic rocks must be the result of a variable source enrichment by slab-derived fluids overprinting a variable depleted mantle wedge. The plateau basalts are less depleted in HFSE and show a more fractionated HREE pattern. These lavas could either result from a slab component or the addition of an OIB-type enriched mantle in their source. (C) 2000 Elsevier Science B.V. All rights reserved.
\end{abstract}

Keywords: Kamchatka; Bakening volcano; Quaternary activity; geochemical evolution; mantle source; fractionation; fluid enrichment; geochemistry

\footnotetext{
* Corresponding author. Tel.: +49-551-393971/72; fax: +49551-393-982.

E-mail address: gwoerne@gwdg.de (G. Wörner).
}

\section{Introduction}

Arc volcanism on Kamchatka comprises some of the most active volcanoes in the world. From E to W 
three major zones are observed: (1) the Eastern volcanic front (EVF); (2) the Central Kamchatka depression with the famous Kluchevskaya Group (CKD); and (3) the western volcanic chain of the Sredinny range (SR). The geology and stratigraphy of many of the volcanoes are known, but compared to other arcs, modern geochemical data are still relatively scarce and the majority are of more regional aspect (Kersting and Arculus, 1995; Hochstaedter et al., 1996; Kepezhinskas et al., 1997; Turner et al., 1998; Churikova et al., 2000; Dorendorf et al., 2000).

Bakening volcano $(2277 \mathrm{~m})$ is one of the least known centers in Kamchatka. This Late PleistoceneHolocene center is located $60 \mathrm{~km}$ to the NW of the active volcanic front and $100 \mathrm{~km} \mathrm{~N}$ of Petropavlovsk. It is thus located between the CKD and the EVF; comprising Avachinsky, Koryaksky, Zupanovsky and Semyachik volcanoes.

Until recently, Bakening volcano was mainly geomorphologically and petrographically studied (Piip, 1947; Svjatlowsky, 1956; Zjurupa, 1978; Melekestsev et al., 1998). Bakening volcano has received increased interest, because Dirksen and Melekestsev (1998) suggested that the most recent eruptions are only about 600-1200 years old, indicating that the Bakening volcano can be regarded as potentially active. This paper provides the first systematic geochemical investigation of this remote volcano including its surrounding monogenetic centers. We use major and trace elements as well as $\mathrm{Sr}$ - and $\mathrm{Nd}$ isotopes, to examine how the volcanism has evolved in this region and to define variable sources in the mantle wedge of the Kamachatka subduction zone beneath the Bakening volcano.

\section{Analytical procedures}

Major and some trace elements $(\mathrm{Ba}, \mathrm{Co}, \mathrm{Cr}, \mathrm{Ga}, \mathrm{Ni}$, $\mathrm{Rb}, \mathrm{Sc}, \mathrm{Sr}, \mathrm{V}, \mathrm{Y}, \mathrm{Zn}, \mathrm{Zr}$ ) were measured on glass fusion discs by XRF analysis. The $2 \sigma$-error for major elements is $<1 \%$ (except $\mathrm{FeO}$ and $\mathrm{Na}_{2} \mathrm{O}$, which are $<2 \%$ ), for trace elements $<5 \%$ (until the limit of $20-30 \mathrm{ppm}$ ). The error of determination of the loss on ignition (LOI) is around 10\%. All other trace elements were determined by ICPMS, with errors $(2 \sigma)<20 \%$ for $\mathrm{Nb}$ and $\mathrm{Ta},<10 \%$ for $\mathrm{Be}$, $\mathrm{Cs}, \mathrm{Cu}, \mathrm{Hf}, \mathrm{Li}, \mathrm{Ni}, \mathrm{Sc}, \mathrm{Y}, \mathrm{Pb}, \mathrm{Th}, \mathrm{Tl}$ and $\mathrm{U}$ and $\sim 5 \%$ for $\mathrm{Rb}, \mathrm{Sr}$ and the REE. The reproducibility was checked by analysis of international standards (JB3, JA2). Some trace elements were measured by both (XRF and ICPMS) methods, the deviation of values is inside the reported errors. Results obtained by XRF were generally preferred for elements with concentrations commonly $>20 \mathrm{ppm}$. The isotope ratios of $\mathrm{Sr}, \mathrm{Nd}$ and $\mathrm{Pb}$ were measured on a Finnigan MAT 252 in Göttingen. Sr and Nd isotope ratios were corrected for mass-fractionation to ${ }^{86} \mathrm{Sr} /{ }^{88} \mathrm{Sr}=0.1194$ and ${ }^{144} \mathrm{Nd} /{ }^{146} \mathrm{Nd}=0.7219$ and referenced to NBS987 (0.710245) for $\mathrm{Sr}$ and La Jolla (0.511847) for $\mathrm{Nd}$ isotopes. Measured values of these standards over the period of the study were $0.710262 \pm 24$ and $0.511847 \pm 20$, respectively. Lead isotopes were corrected to NBS981 (Todt et al., 1984). Thirteen measurements of this standard gave a mean of ${ }^{206} \mathrm{~Pb} /{ }^{204} \mathrm{~Pb}=16.90 \pm 0.01, \quad{ }^{206} \mathrm{~Pb} /{ }^{204} \mathrm{~Pb}=15.44 \pm$ 0.02 and ${ }^{206} \mathrm{~Pb} /{ }^{204} \mathrm{~Pb}=37.53 \pm 0.05$. Blanks for $\mathrm{Sr}$, $\mathrm{Nd}$ and $\mathrm{Pb}$ were $<1,<0.03$ and $<0.5 \mathrm{ng}$, respectively; which are insignificant. From continuous measurement of standards and duplicate measurements of samples, relative $2 \sigma$-errors less than $0.004 \%$ for $\mathrm{Sr}$ and $\mathrm{Nd}$, and less than $0.1 \%$ for $\mathrm{Pb}$ were determined.

\section{Volcanic geology and erupted rock types}

The basement in the Bakening region is formed by Miocene volcano-sedimentary rocks of the Ganalsky Ridge. Lower Cretaceous sedimentary rocks and Miocene intrusive rocks are exposed at the uplifted E border of the Srednaya Avacha Valley. Lower Quaternary plateau basalts and andesites can be found in the $\mathrm{N}$ and SE of Bakening. These rock units are bordered by Lower to Middle Pleistocene $\mathrm{N}-\mathrm{S}$ trending faults. Bakening stratovolcano developed in the Srednaya Avacha depression where a maximum vertical displacement up to $2500 \mathrm{~m}$ has been estimated by Zjurupa (1978). This old fault system is crossed by a NNE-SSW-directed Late Pleistocene fault system. These younger faults are obviously connected with the same stress field that also formed the Central Kamchatka depression.

Quaternary volcanism in the studied area is bimodal. Andesitic to dacitic rocks form the Bakening stratovolcano and some adjacent cones nearby. 
Monogenetic cinder cones are developed at the intersection of the two fault systems and characterized by mainly basaltic volcanism. Both suites erupted closely in space and time.

\subsection{Bakening volcano main cone}

Bakening volcano $(2277 \mathrm{~m})$ is $5 \mathrm{~km}$ wide in basal diameter and rises around $1500 \mathrm{~m}$ above its surroundings (Fig. 1). From the west the cone appears relatively well preserved. The internal structure of the stratovolcano is best studied on the SE side, where a sector collapse has exposed deeper sections. This collapse produced a debris avalanche, dated at 8000-8500 years BP $\left({ }^{14} \mathrm{C}\right)$ (Melekestsev et al., 1998). The Tertiary volcanic and volcaniclastic basement rocks are strongly altered. This alteration is probably connected with the intrusion of Bakening andesites. Less-altered Tertiary rocks can be found in the south and west of the volcano. A great number of Quaternary andesitic and dacitic intrusions and block lava flows up to $1000 \mathrm{~m}$ thick are piled up on this basement. In the upper part of the volcano the remains of the former crater are preserved and documented by strongly altered crater fill. Numerous dikes are eroded out the slopes by erosion.

Two suites of rocks at Bakening stratovolcano are distinguished: The older Bakening rocks are dacitic extrusions, exposed on the S and SW flanks between 1300 and $1700 \mathrm{~m}$. These rocks are light gray, but often oxidized to a reddish color. They contain around 15$20 \%$ plagioclase, $3-5 \%$ amphibole and $1 \%$ magnetite in a fine-grained groundmass. Amphibole crystals are long prismatic and often show opacite rims. They also occur in cumulates together with plagioclase, magnetite and glass.

The second younger series is composed of pyroxene andesite lava flows, which cover the whole volcano and form the dikes. These relatively well-preserved dark rocks are amphibole-free and contain 25-30\% plagioclase, 5-10\% dark green ortho- and clinopyroxene, $1 \%$ magnetite and sometimes relicts of yellow-green olivine in a glassy to fine-grained groundmass. Olivine grains are partly reacted to orthopyroxene and magnetite. Phenocrysts are small, generally about $1 \mathrm{~mm}$, rarely up to $5 \mathrm{~mm}$. At the $\mathrm{E}$ slope of Bakening red tuffs are observed, which belong to the young pyroxene andesite suite.
Bakening lavas frequently contain xenoliths of sedimentary and volcanic rocks from the basement and older Bakening eruptions and cumulates.

Activity of Bakening stratovolcano presumably began in Late Pleistocene times. Some flows are clearly Holocene, the youngest eruptions at the SE slope are only 3500 years old (Melekestsev et al., 1998).

\subsection{Novy Bakening and comparable andesitic to dacitic cones}

Bakening stratovolcano is surrounded within $5 \mathrm{~km}$ by small andesitic to dacitic cones (Fig. 1). Novy Bakening, situated in the NNE of the stratovolcano, is the largest, youngest and best preserved. It is composed of eight dacitic flows, flowing in $\mathrm{E}$ and $\mathrm{W}$ directions from the $250 \mathrm{~m}$ high eruption center. The lava contains around $10 \%$ plagioclase and lessfrequently pyroxene (up to $2 \%$ ) and amphibole (up to $4 \%$ ). The lower flows are slightly more amphibole rich compared to the upper flows but do not contain pyroxene. Two similar small extrusions just to the $\mathrm{N}$ suggest, that the eruption started from a nearly $\mathrm{N}-\mathrm{S}$ directed fissure and concentrated later on Novy Bakening. The largest $5 \mathrm{~km}$ long flow was erupted to the $\mathrm{W}$ into a glacier valley. The small extrusion at Lake Vysokoe W of Bakening is similar in mineral composition to Novy Bakening rocks (7\% plagioclase, $3 \%$ amphibole). The cones SW of Bakening at the Malye Lakes contain plagioclase $(20 \%)$ and low amounts of pyroxene (1-2\%) and amphibole (2-3\%) and are similar to the older extrusions at Bakening main cone. These flows are overlain by the younger pyroxene-andesite flows of Bakening.

\subsection{Basaltic monogenetic centers}

Basaltic monogenetic volcanic centers occur W of Bakening, at Srednaya Avacha river and its right tributary Kostakan river, in the Kavyche valley, and around $30 \mathrm{~km}$ to the $\mathrm{E}$ in the Levaya Avacha Valley (Fig. 1). These centers form small scoria cones, which were the source of lava flows several $\mathrm{km}$ in extension. The eruption age is deduced from morphological preservation and tephrochronology to be Late Pleistocene-Holocene. The ages of the youngest cones are 600-1200 ${ }^{14} \mathrm{C}$ years BP (Dirksen and Melekestsev, 1998). The crystallization sequence is 


\section{Geological Map of Bakening Volcano and Surroundings}

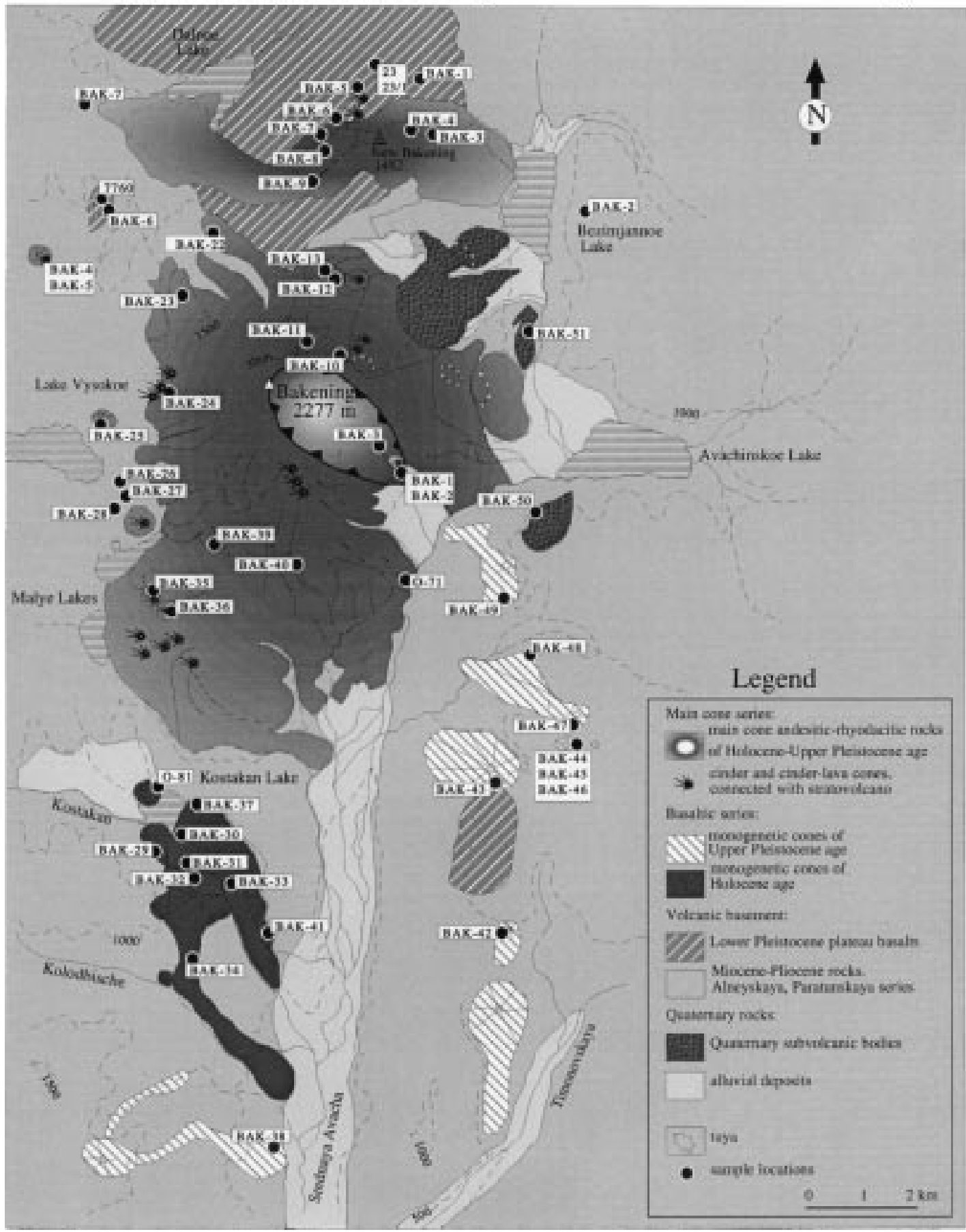

Fig. 1. Geological map of the Bakening volcano and the surroundings based on field studies in 1995 and 1996 and an unpublished map of I. Melekestsev. Sampled points are indicated, 12 additional scattered sampling sites of mafic monogenetic centers are outside the map area towards the NE and $\mathrm{E}$. 
olivine $\rightarrow$ olivine + clinopyroxene $\rightarrow$ olivine + clinopyroxene + plagioclase $\rightarrow$ plagioclase. Olivine, up to $1 \mathrm{~mm}$ in size, contains Cr-spinel inclusions and often shows skeletal growth. Clinopyroxene is absent or less frequent than olivine except for samples from the pass between Srednaya Avacha and Kavyche valley. Clinopyroxenes in these samples are elongated and show hour-glass structures.

\subsection{Rhyodacitic monogenetic centers}

We sampled subglacial rhyodacitic extrusions, which form so-called tuyas and are up to $500 \mathrm{~m}$ in diameter. These occur at the NW base of Bakening, at the Avachinskoe lake and in the Kavyche valley. Similar highly differentiated rocks also occur further to the N at the NW slope of the Ganalsky Ridge. They are formed by chilled volcanic rocks and contain plagioclase and minor amounts of biotite and clinopyroxene in a fine-grained groundmass. The black obsidian from the tuya at Kavyche River is phenocryst-free.

\subsection{Plateau basalts}

To the N and SE of Bakening, in the Srednaya Avacha and the Kavyche valleys remnants of plateau-forming basalts and basaltic andesites are exposed. These "plateau basalts" were erupted all over Kamchatka from the Miocene to Lower Quaternary. There are no detailed studies of the eruption centers, eruption rate and duration of plateau volcanism. Some age data is given by Kepezhinskas et al. (1997), which distinguish a tilted Older Series $(\sim 14 \mathrm{Ma})$ from a subhorizontal Younger Series (2.6-3.0 Ma). Our samples belong entirely to the Younger Series, which is equivalent to the $\mathrm{K}-\mathrm{Na}-$ alkali-olivine basalts, described by Volynets (1994). Older and glacially dissected Lower Pleistocene plateau basalts occur from 800 to $1500 \mathrm{~m}$ above sea level and form the base of Novy Bakening and of Bakening volcano except at its southern margins. Single lava flows are tens of meters thick and extend over kilometers. Our samples are derived from locations $\mathrm{N}$ and NNW of Bakening volcano (Fig. 1). The mineral mode is similar to the Quaternary basalts but phenocrysts are less frequent and smaller in size.

One flow NNW of Novy Bakening is particularly rich in xenoliths (dunites, harzburgites, green and black pyroxenites). These xenoliths are derived from the transition zone between the plagioclase and spinel stability fields in the lithospheric mantle (Koloskov et al., 1997) below Bakening. For some of these xenoliths Kepezhinskas and Defant (1996) propose an enrichment by carbonate-rich melts derived from a slab melt.

\section{Geochemistry}

The entire range of Quaternary rock types at Bakening volcano was sampled. Sixty samples were analyzed for major and some trace elements by XRF (Table 1), a selection of 43 samples for trace elements by ICPMS. A representative suite (12 samples) for Srand $\mathrm{Nd}$-isotopes and only six samples were analyzed for $\mathrm{Pb}$-isotopes.

\subsection{Major elements}

Bulk compositions range from basalts to rhyodacites. All samples are fresh and have low LOI $(<1 \%)$ and low $\mathrm{Fe}_{2} \mathrm{O}_{3} / \mathrm{FeO}$ ratios $(<0.3-0.6)$. The plateau basalts have slightly higher $\mathrm{Fe}_{2} \mathrm{O}_{3} / \mathrm{FeO}$ ratios between $0.4-0.8$ probably from minor alteration of these older rocks. Dacite extrusions are slightly more oxidized up to $\mathrm{Fe}_{2} \mathrm{O}_{3} / \mathrm{FeO}$ ratios of 1.0. No correlation between LOI and degree of oxidization or major and trace element contents exists. All samples follow a calc-alkaline trend (Fig. 2), except some of the most mafic plateau basalts which are slightly more alkaline.

Pleistocene and Holocene mafic monogenetic centers range from high-Mg basalts with $49 \% \mathrm{SiO}_{2}$, $10 \% \mathrm{MgO}$ and $16 \% \mathrm{Al}_{2} \mathrm{O}_{3}$ to high- $\mathrm{Al}$ basaltic andesites with $56 \% \mathrm{SiO}_{2}, 4 \% \mathrm{MgO}$ and $20 \% \mathrm{Al}_{2} \mathrm{O}_{3}$ (Fig. $3)$. The decrease in $\mathrm{MgO}$ is coupled with a decrease in $\mathrm{FeO}, \mathrm{CaO}$ and $\mathrm{TiO}_{2}$ and an increase in $\mathrm{SiO}_{2}, \mathrm{Na}_{2} \mathrm{O}$, $\mathrm{Al}_{2} \mathrm{O}_{3}$ and $\mathrm{P}_{2} \mathrm{O}_{5}$ (Fig. 3). No systematic variations are observed for $\mathrm{MnO}$ and $\mathrm{K}_{2} \mathrm{O}$. Two slightly alkaline basaltic samples (O35, BAK64) show higher $\mathrm{TiO}_{2}$, $\mathrm{Na}_{2} \mathrm{O}$ and $\mathrm{P}_{2} \mathrm{O}_{5}$ contents. There exists no obvious relationship between the geochemistry and sequence of eruptions of the monogenetic centers.

The older Plateau basalts differ significantly from the younger basaltic centers by their higher concentrations of $\mathrm{TiO}_{2}, \mathrm{~K}_{2} \mathrm{O}, \mathrm{Na}_{2} \mathrm{O}$ and $\mathrm{P}_{2} \mathrm{O}_{5}$, and lower concentrations of $\mathrm{Al}_{2} \mathrm{O}_{3}$ and $\mathrm{CaO}$ (at a given $\mathrm{MgO}$ ). 
Table 1

Major (wt.\%) and trace element (ppm) analyses of Quaternary volcanics in the Bakening area

Basalts and basaltic andesites from monogenetic centers

Levaya Avacha Valley $\quad$ Kostakan

BAK04 BAK24 BAK61 BAK62 BAK49 BAK43 BAK44 BAK45 BAK47 BAK64 BAK42 BAK31 BAK37 BAK30 BAK34

\begin{tabular}{|c|c|c|c|c|c|c|c|c|c|c|c|c|c|c|c|}
\hline $\mathrm{SiO}_{2}$ & 48.75 & 49.68 & 50.40 & 50.40 & 51.41 & 51.70 & 52.08 & 52.46 & 52.63 & 53.30 & 53.55 & 50.26 & 50.37 & 50.53 & 51.16 \\
\hline $\mathrm{TiO}_{2}$ & 1.13 & 1.03 & 1.06 & 1.07 & 0.90 & 0.91 & 1.05 & 0.89 & 0.89 & 1.36 & 0.98 & 1.09 & 1.09 & 1.08 & 1.04 \\
\hline $\mathrm{Al}_{2} \mathrm{O}_{3}$ & 17.26 & 16.22 & 16.70 & 16.65 & 17.80 & 18.43 & 17.07 & 19.22 & 19.33 & 16.81 & 16.72 & 16.10 & 16.30 & 16.29 & 16.88 \\
\hline $\mathrm{Fe}_{2} \mathrm{O}_{3}$ & 2.68 & 2.46 & 3.00 & 2.83 & 2.59 & 2.21 & 2.72 & 2.77 & 3.06 & 3.39 & 2.48 & 2.24 & 2.26 & 2.58 & 2.08 \\
\hline $\mathrm{FeO}$ & 7.09 & 6.91 & 6.25 & 6.45 & 6.19 & 6.50 & 5.92 & 5.95 & 5.80 & 5.63 & 5.35 & 6.89 & 6.86 & 6.56 & 6.70 \\
\hline $\mathrm{MnO}$ & 0.17 & 0.17 & 0.16 & 0.17 & 0.17 & 0.16 & 0.15 & 0.17 & 0.17 & 0.15 & 0.14 & 0.17 & 0.17 & 0.17 & 0.17 \\
\hline $\mathrm{MgO}$ & 8.09 & 9.83 & 8.51 & 8.61 & 6.63 & 5.74 & 7.47 & 4.59 & 4.65 & 6.02 & 7.28 & 9.15 & 8.84 & 9.05 & 7.80 \\
\hline $\mathrm{CaO}$ & 10.47 & 9.67 & 9.07 & 9.09 & 8.82 & 8.77 & 8.51 & 8.36 & 8.45 & 7.80 & 7.92 & 9.32 & 9.27 & 9.22 & 8.94 \\
\hline $\mathrm{Na}_{2} \mathrm{O}$ & 2.83 & 2.87 & 3.16 & 3.20 & 3.27 & 3.32 & 3.48 & 3.75 & 3.69 & 3.96 & 3.70 & 3.21 & 3.15 & 3.16 & 3.18 \\
\hline $\mathrm{K}_{2} \mathrm{O}$ & 0.70 & 0.70 & 0.89 & 0.91 & 0.67 & 0.90 & 0.91 & 0.87 & 0.86 & 1.07 & 1.04 & 0.95 & 0.95 & 0.94 & 0.91 \\
\hline $\mathrm{P}_{2} \mathrm{O}_{5}$ & 0.17 & 0.21 & 0.23 & 0.23 & 0.23 & 0.25 & 0.26 & 0.31 & 0.31 & 0.42 & 0.29 & 0.26 & 0.26 & 0.26 & 0.23 \\
\hline LOI & 0.25 & 0.60 & 0.56 & 0.46 & 0.54 & 0.49 & 0.16 & 0.47 & 0.17 & 0.30 & 0.36 & 0.27 & 0.20 & 0.32 & 0.47 \\
\hline Total & 99.58 & 100.34 & 100.00 & 100.06 & 99.21 & 99.38 & 99.77 & 99.81 & 100.00 & 100.22 & 99.81 & 99.89 & 99.71 & 100.15 & 99.55 \\
\hline $\mathrm{Li}$ & 8.2 & 6.0 & & & 7.5 & 8.2 & 6.9 & 7.8 & 6.9 & & 5.7 & 8.5 & 6.1 & 8.0 & 8.1 \\
\hline $\mathrm{Be}$ & 0.74 & 0.60 & & & 0.74 & 0.73 & 0.90 & 0.70 & 0.72 & & 0.99 & 0.80 & 0.76 & 0.80 & 0.78 \\
\hline $\mathrm{Sc}$ & 38 & 34 & 30 & 26 & 24 & 25 & 23 & 19 & 19 & 20 & 25 & 30 & 30 & 30 & 29 \\
\hline $\mathrm{V}$ & 304 & 261 & 255 & 249 & 207 & 217 & 223 & 181 & 186 & 220 & 205 & 253 & 254 & 249 & 237 \\
\hline $\mathrm{Cr}$ & 233 & 494 & 329 & 337 & 179 & 91 & 285 & 28 & 26 & 217 & 292 & 394 & 360 & 377 & 272 \\
\hline Co & 38 & 46 & 39 & 42 & 31 & 33 & 36 & 26 & 29 & 34 & 34 & 44 & 41 & 43 & 38 \\
\hline $\mathrm{Ni}$ & 84 & 169 & 141 & 170 & 69 & 46 & 126 & 17 & 25 & 75 & 122 & 155 & 152 & 155 & 117 \\
\hline $\mathrm{Cu}$ & 63 & 63 & & & 48 & 43 & 52 & 41 & 27 & & 25 & 68 & 64 & 63 & 58 \\
\hline $\mathrm{Zn}$ & 69 & 77 & 81 & 80 & 70 & 75 & 74 & 84 & 82 & 100 & 80 & 76 & 76 & 76 & 74 \\
\hline $\mathrm{Ga}$ & 16 & 17 & 20 & 16 & 14 & 16 & 17 & 18 & 19 & 20 & 17 & 17 & 17 & 17 & 17 \\
\hline $\mathrm{Rb}$ & 27 & 15 & 14 & 18 & 13 & 18 & 18 & 16 & 15 & 15 & 20 & 22 & 25 & 19 & 20 \\
\hline $\mathrm{Sr}$ & 429 & 423 & 496 & 494 & 526 & 536 & 525 & 628 & 627 & 708 & 563 & 505 & 512 & 497 & 471 \\
\hline $\mathrm{Y}$ & 10 & 21 & 16 & 20 & 21 & 21 & 22 & 23 & 20 & 22 & 18 & 21 & 20 & 22 & 24 \\
\hline $\mathrm{Zr}$ & 64 & 82 & 88 & 86 & 94 & 84 & 102 & 90 & 91 & 125 & 107 & 91 & 91 & 90 & 92 \\
\hline $\mathrm{Nb}$ & 2.8 & 2.8 & & & 2.7 & 2.7 & 3.6 & 2.8 & 2.7 & & 3.6 & 4.3 & 3.4 & 3.1 & 3.6 \\
\hline Cs & 0.38 & 0.49 & & & 0.43 & 0.62 & 0.30 & 0.54 & 0.41 & & 0.54 & 0.76 & 0.71 & 0.70 & 0.58 \\
\hline $\mathrm{Ba}$ & 223 & 241 & 313 & 318 & 275 & 412 & 358 & 394 & 401 & 345 & 375 & 400 & 382 & 379 & 304 \\
\hline $\mathrm{La}$ & 7.1 & 7.6 & & & 7.9 & 9.0 & 10.2 & 9.4 & 9.4 & & 11.6 & 10.7 & 10.5 & 10.0 & 9.4 \\
\hline $\mathrm{Ce}$ & 18.2 & 19.4 & & & 20.1 & 21.9 & 24.6 & 23.3 & 23.3 & & 28.3 & 24.7 & 24.7 & 23.4 & 22.7 \\
\hline $\operatorname{Pr}$ & 2.9 & 3.0 & & & 3.2 & 3.3 & 3.7 & 3.6 & 3.6 & & 4.3 & 3.6 & 3.6 & 3.5 & 3.4 \\
\hline $\mathrm{Nd}$ & 13.7 & 13.8 & & & 14.8 & 15.5 & 17.3 & 16.6 & 16.7 & & 19.3 & 16.7 & 16.6 & 16.2 & 15.7 \\
\hline $\mathrm{Sm}$ & 3.6 & 3.7 & & & 3.8 & 4.0 & 4.3 & 4.3 & 4.2 & & 4.6 & 4.3 & 4.3 & 4.1 & 4.0 \\
\hline $\mathrm{Eu}$ & 1.13 & 1.15 & & & 1.18 & 1.20 & 1.32 & 1.29 & 1.32 & & 1.36 & 1.29 & 1.28 & 1.25 & 1.24 \\
\hline Gd & 3.2 & 3.4 & & & 3.2 & 3.6 & 3.9 & 3.7 & 3.6 & & 3.9 & 3.8 & 3.9 & 3.8 & 3.7 \\
\hline $\mathrm{Tb}$ & 0.53 & 0.54 & & & 0.53 & 0.58 & 0.62 & 0.58 & 0.58 & & 0.60 & 0.61 & 0.62 & 0.61 & 0.61 \\
\hline Dy & 3.1 & 3.3 & & & 3.1 & 3.4 & 3.6 & 3.4 & 3.4 & & 3.3 & 3.6 & 3.7 & 3.6 & 3.6 \\
\hline Ho & 0.64 & 0.67 & & & 0.62 & 0.69 & 0.70 & 0.68 & 0.68 & & 0.63 & 0.70 & 0.72 & 0.71 & 0.72 \\
\hline $\mathrm{Er}$ & 1.81 & 1.88 & & & 1.82 & 2.04 & 1.93 & 2.01 & 2.03 & & 1.83 & 1.99 & 2.07 & 2.01 & 2.08 \\
\hline $\mathrm{Tm}$ & 0.27 & 0.27 & & & 0.27 & 0.31 & 0.29 & 0.30 & 0.31 & & 0.26 & 0.28 & 0.29 & 0.29 & 0.31 \\
\hline $\mathrm{Yb}$ & 1.80 & 1.81 & & & 1.76 & 1.92 & 1.81 & 1.96 & 1.95 & & 1.72 & 1.85 & 1.88 & 1.89 & 1.96 \\
\hline $\mathrm{Lu}$ & 0.27 & 0.26 & & & 0.26 & 0.30 & 0.26 & 0.31 & 0.31 & & 0.25 & 0.28 & 0.28 & 0.28 & 0.30 \\
\hline Hf & 1.85 & 2.08 & & & 2.09 & 2.21 & 2.60 & 2.35 & 2.33 & & 2.77 & 2.37 & 2.44 & 2.35 & 2.35 \\
\hline $\mathrm{Ta}$ & 0.18 & 0.33 & & & 0.18 & 0.22 & 0.21 & 0.18 & 0.20 & & 0.18 & 0.27 & 0.18 & 0.15 & 0.20 \\
\hline $\mathrm{Tl}$ & 0.05 & 0.04 & & & 0.06 & 0.01 & 0.05 & 0.04 & 0.05 & & 0.05 & 0.06 & 0.05 & 0.07 & 0.09 \\
\hline $\mathrm{Pb}$ & 2.1 & 2.3 & & & 2.5 & 2.9 & 2.9 & 3.3 & 2.9 & & 2.7 & 3.5 & 3.8 & 3.5 & 2.9 \\
\hline Th & 0.67 & 0.89 & & & 0.75 & 1.45 & 1.41 & 1.20 & 1.19 & & 1.34 & 1.60 & 1.75 & 1.49 & 1.46 \\
\hline $\mathrm{U}$ & 0.33 & 0.42 & & & 0.35 & 0.58 & 0.61 & 0.52 & 0.54 & & 0.62 & 0.66 & 0.67 & 0.64 & 0.56 \\
\hline
\end{tabular}


Table 1 (continued)

\begin{tabular}{|c|c|c|c|c|c|c|c|c|c|c|c|c|c|c|c|}
\hline \multirow{2}{*}{\multicolumn{3}{|c|}{ Kostakan }} & \multirow{2}{*}{\multicolumn{3}{|c|}{ Mount Peschanaja }} & \multicolumn{10}{|c|}{ Basalts and basaltic andesites from monogenetic centers } \\
\hline & & & & & & \multicolumn{10}{|c|}{ Kavycha Valley } \\
\hline BAK32 & BAK33 & BAK41 & $\mathrm{O}-40$ & O-26 & O-35 & BAK21A & BAK15 & BAK14 & BAK17 & BAK18 & BAK20A & BAK20B & BAK16 & BAK38 & BAK21B \\
\hline 51.23 & 51.32 & 51.50 & 49.77 & 50.41 & 51.49 & 50.07 & 50.13 & 50.32 & 51.23 & 53.50 & 53.51 & 53.61 & 54.41 & 54.53 & 55.08 \\
\hline 1.02 & 1.03 & 1.02 & 1.20 & 1.10 & 1.29 & 0.97 & 0.96 & 0.99 & 1.15 & 0.79 & 0.94 & 0.93 & 0.94 & 0.86 & 0.82 \\
\hline 16.70 & 16.76 & 16.99 & 16.84 & 16.28 & 16.70 & 16.85 & 16.80 & 16.41 & 16.30 & 19.44 & 18.88 & 18.94 & 17.41 & 19.12 & 18.30 \\
\hline 1.93 & 2.35 & 1.91 & 3.10 & 2.53 & 3.00 & 3.34 & 2.60 & 2.18 & 2.01 & 2.34 & 3.11 & 2.38 & 2.41 & 3.26 & 1.94 \\
\hline 6.86 & 6.56 & 6.74 & 6.12 & 6.42 & 5.52 & 6.10 & 6.73 & 7.02 & 6.52 & 6.13 & 5.21 & 5.92 & 5.58 & 5.08 & 5.92 \\
\hline 0.17 & 0.17 & 0.17 & 0.16 & 0.15 & 0.15 & 0.19 & 0.19 & 0.18 & 0.16 & 0.17 & 0.15 & 0.15 & 0.16 & 0.17 & 0.15 \\
\hline 8.09 & 8.32 & 7.56 & 8.61 & 9.40 & 6.72 & 8.24 & 8.33 & 8.92 & 8.29 & 3.93 & 4.57 & 4.50 & 5.32 & 3.85 & 3.75 \\
\hline 9.01 & 9.06 & 8.91 & 9.60 & 9.06 & 9.52 & 9.47 & 9.51 & 9.74 & 8.90 & 8.27 & 8.25 & 8.29 & 8.48 & 7.66 & 7.20 \\
\hline 3.16 & 3.14 & 3.21 & 3.24 & 3.22 & 3.56 & 2.91 & 3.00 & 3.00 & 3.38 & 3.75 & 3.68 & 3.68 & 3.46 & 3.87 & 3.80 \\
\hline 0.91 & 0.92 & 0.92 & 0.88 & 0.90 & 1.22 & 0.75 & 0.77 & 0.68 & 1.01 & 0.81 & 0.90 & 0.90 & 1.13 & 0.96 & 1.20 \\
\hline 0.23 & 0.23 & 0.23 & 0.24 & 0.28 & 0.41 & 0.26 & 0.26 & 0.23 & 0.29 & 0.29 & 0.23 & 0.24 & 0.21 & 0.25 & 0.24 \\
\hline 0.49 & 0.43 & 0.41 & 0.31 & 0.23 & 0.53 & 0.21 & 0.51 & 0.49 & 0.60 & 0.45 & 0.75 & 0.77 & 0.66 & 0.71 & 0.44 \\
\hline \multirow[t]{3}{*}{99.81} & 100.27 & 99.57 & 100.04 & 99.96 & 100.09 & 99.36 & 99.78 & 100.15 & 99.83 & 99.86 & 100.18 & 100.29 & 100.14 & 100.30 & 98.84 \\
\hline & 7.5 & 7.5 & 6.8 & 7.2 & 8.1 & & 7.1 & 6.2 & 7.4 & 8.1 & & 6.1 & 9.6 & 8.9 & \\
\hline & 0.77 & 0.74 & 0.70 & 0.69 & 0.82 & & 0.82 & 0.71 & 0.86 & 0.81 & & 0.76 & 0.76 & 0.75 & \\
\hline 29 & 29 & 26 & 31 & 30 & 29 & 29 & 32 & 33 & 29 & 17 & 20 & 20 & 27 & 16 & 21 \\
\hline 242 & 240 & 238 & 285 & 250 & 275 & 230 & 235 & 242 & 236 & 158 & 201 & 209 & 235 & 181 & 171 \\
\hline 301 & 315 & 259 & 283 & 343 & 165 & 311 & 317 & 411 & 349 & 15 & 29 & 30 & 104 & 15 & 15 \\
\hline 41 & 43 & 38 & 44 & 43 & 35 & 38 & 40 & 41 & 38 & 27 & 28 & 29 & 32 & 24 & 25 \\
\hline \multirow[t]{2}{*}{125} & 134 & 111 & 127 & 167 & 62 & 117 & 121 & 126 & 130 & 11 & 17 & 18 & 32 & 6 & 0 \\
\hline & 59 & 52 & 52 & 40 & 80 & & 55 & 60 & 52 & 37 & & 56 & 64 & 38 & \\
\hline 73 & 74 & 73 & 71 & 71 & 75 & 78 & 80 & 77 & 75 & 88 & 78 & 79 & 77 & 77 & 70 \\
\hline 17 & 17 & 17 & 17 & 17 & 17 & 18 & 17 & 17 & 16 & 19 & 19 & 18 & 18 & 19 & 18 \\
\hline 17 & 19 & 18 & 12 & 17 & 21 & 11 & 21 & 17 & 21 & 17 & 15 & 15 & 22 & 15 & 17 \\
\hline 468 & 463 & 475 & 518 & 547 & 706 & 514 & 531 & 453 & 529 & 628 & 605 & 609 & 481 & 596 & 567 \\
\hline 23 & 21 & 22 & 23 & 20 & 32 & 3 & 24 & 24 & 23 & 22 & 19 & 21 & 21 & 18 & 23 \\
\hline \multirow[t]{3}{*}{90} & 91 & 92 & 92 & 92 & 115 & 101 & 96 & 96 & 108 & 93 & 92 & 90 & 85 & 86 & 99 \\
\hline & 3.6 & 3.2 & 3.5 & 3.5 & 5.1 & & 2.3 & 3.0 & 4.7 & 2.8 & & 2.4 & 3.1 & 3.1 & \\
\hline & 0.64 & 0.60 & 0.57 & 0.29 & 0.62 & & 0.59 & 0.45 & 0.64 & 0.45 & & 0.47 & 0.67 & 0.49 & \\
\hline \multirow[t]{21}{*}{313} & 312 & 298 & 326 & 340 & 440 & 184 & 194 & 185 & 346 & 328 & 335 & 336 & 358 & 336 & 375 \\
\hline & 9.6 & 9.4 & 9.1 & 10.1 & 14.7 & & 8.9 & 8.1 & 11.0 & 9.6 & & 9.8 & 9.9 & 9.5 & \\
\hline & 23.0 & 22.6 & 21.9 & 23.8 & 36.7 & & 23.2 & 21.5 & 26.7 & 23.7 & & 23.5 & 23.3 & 22.6 & \\
\hline & 3.4 & 3.4 & 3.3 & 3.6 & 5.7 & & 3.7 & 3.4 & 4.0 & 3.6 & & 3.5 & 3.4 & 3.4 & \\
\hline & 15.9 & 15.6 & 15.8 & 16.6 & 25.5 & & 17.4 & 15.7 & 18.6 & 16.4 & & 16.1 & 15.3 & 15.5 & \\
\hline & 4.1 & 4.0 & 4.1 & 4.1 & 5.7 & & 4.5 & 4.1 & 4.7 & 4.1 & & 4.0 & 3.9 & 3.9 & \\
\hline & 1.28 & 1.22 & 1.30 & 1.28 & 1.64 & & 1.32 & 1.28 & 1.39 & 1.26 & & 1.22 & 1.16 & 1.21 & \\
\hline & 3.8 & 3.7 & 3.8 & 3.7 & 4.4 & & 4.0 & 3.8 & 4.1 & 3.4 & & 3.4 & 3.4 & 3.3 & \\
\hline & 0.62 & 0.61 & 0.61 & 0.59 & 0.64 & & 0.66 & 0.64 & 0.65 & 0.56 & & 0.54 & 0.56 & 0.54 & \\
\hline & 3.7 & 3.6 & 3.6 & 3.4 & 3.6 & & 4.0 & 4.0 & 3.8 & 3.2 & & 3.2 & 3.3 & 3.1 & \\
\hline & 0.74 & 0.73 & 0.72 & 0.66 & 0.68 & & 0.81 & 0.83 & 0.75 & 0.63 & & 0.64 & 0.68 & 0.63 & \\
\hline & 2.10 & 2.12 & 2.10 & 1.91 & 1.88 & & 2.34 & 2.38 & 2.17 & 1.91 & & 1.87 & 1.89 & 1.91 & \\
\hline & 0.31 & 0.31 & 0.30 & 0.28 & 0.26 & & 0.35 & 0.36 & 0.32 & 0.29 & & 0.28 & 0.28 & 0.28 & \\
\hline & 2.03 & 1.98 & 1.89 & 1.79 & 1.69 & & 2.23 & 2.33 & 2.04 & 1.82 & & 1.76 & 1.85 & 1.85 & \\
\hline & 0.30 & 0.29 & 0.29 & 0.26 & 0.25 & & 0.34 & 0.34 & 0.31 & 0.28 & & 0.27 & 0.27 & 0.28 & \\
\hline & 2.46 & 2.42 & 2.33 & 2.38 & 2.84 & & 2.48 & 2.41 & 2.73 & 2.35 & & 2.35 & 2.27 & 2.25 & \\
\hline & 0.23 & 0.20 & 0.23 & 0.22 & 0.29 & & 0.11 & 0.45 & 0.29 & 0.19 & & 0.17 & 0.22 & 0.20 & \\
\hline & 0.06 & 0.08 & 0.03 & 0.03 & 0.09 & & 0.04 & 0.05 & 0.08 & 0.07 & & 0.09 & 0.06 & 0.08 & \\
\hline & 3.2 & 2.9 & 2.8 & 2.8 & 3.7 & & 2.1 & 2.1 & 2.8 & 3.1 & & 3.1 & 3.5 & 3.5 & \\
\hline & 1.76 & 1.53 & 1.26 & 1.24 & 1.30 & & 0.95 & 0.81 & 1.42 & 1.08 & & 1.36 & 1.77 & 1.16 & \\
\hline & 0.60 & 0.54 & 0.55 & 0.55 & 0.61 & & 0.52 & 0.44 & 0.60 & 0.48 & & 0.55 & 0.70 & 0.53 & \\
\hline
\end{tabular}


Table 1 (continued)

\begin{tabular}{|c|c|c|c|c|c|c|c|c|c|c|c|c|c|c|}
\hline & \multirow{2}{*}{\multicolumn{9}{|c|}{$\frac{\text { Plateau basalts }}{\text { Levaya Avacha Valley }}$}} & \multicolumn{5}{|c|}{ Bakening andesites/dacites } \\
\hline & & & & & & & & & & \multicolumn{2}{|c|}{ amph-andesites } & \multicolumn{3}{|c|}{$2 \mathrm{px}$-andesites } \\
\hline & $23 / 7$ & $23 / 8$ & BAK01 & 23 & $23 / 1$ & 121 & 120 & 7760 & BAK06 & BAK10 & BAK12 & BAK22 & BAK39 & BAK23 \\
\hline $\mathrm{SiO}_{2}$ & 48.70 & 49.10 & 49.30 & 49.70 & 49.80 & 50.70 & 51.00 & 51.90 & 53.23 & 62.39 & 64.28 & 58.86 & 59.12 & 60.22 \\
\hline $\mathrm{TiO}_{2}$ & 1.62 & 1.56 & 1.59 & 1.16 & 1.15 & 1.70 & 1.61 & 1.76 & 1.41 & 0.49 & 0.52 & 0.81 & 0.80 & 0.74 \\
\hline $\mathrm{Al}_{2} \mathrm{O}_{3}$ & 16.10 & 16.20 & 16.43 & 15.76 & 15.76 & 15.87 & 16.05 & 16.13 & 15.86 & 17.36 & 17.02 & 17.08 & 16.79 & 17.09 \\
\hline $\mathrm{Fe}_{2} \mathrm{O}_{3}$ & 11.18 & 10.80 & 3.50 & 2.81 & 4.30 & 2.94 & 2.94 & 4.00 & 3.39 & 2.28 & 1.62 & 1.40 & 1.41 & 1.47 \\
\hline $\mathrm{FeO}$ & & & 6.57 & 6.79 & 5.35 & 6.03 & 6.03 & 5.81 & 5.45 & 2.55 & 1.95 & 4.51 & 4.43 & 4.04 \\
\hline $\mathrm{MnO}$ & 0.19 & 0.19 & 0.19 & 0.16 & 0.16 & 0.15 & 0.15 & 0.15 & 0.14 & 0.13 & 0.10 & 0.11 & 0.11 & 0.10 \\
\hline $\mathrm{MgO}$ & 7.80 & 7.84 & 7.54 & 9.30 & 9.17 & 8.84 & 9.03 & 6.47 & 5.77 & 1.90 & 1.13 & 3.51 & 3.67 & 3.32 \\
\hline $\mathrm{CaO}$ & 8.40 & 8.19 & 8.34 & 9.33 & 9.33 & 8.18 & 8.15 & 8.52 & 7.74 & 5.09 & 3.88 & 6.34 & 6.47 & 6.06 \\
\hline $\mathrm{Na}_{2} \mathrm{O}$ & 3.72 & 3.81 & 3.82 & 3.05 & 3.08 & 3.66 & 3.72 & 3.66 & 3.66 & 4.18 & 4.79 & 3.78 & 3.76 & 3.89 \\
\hline $\mathrm{K}_{2} \mathrm{O}$ & 1.68 & 1.73 & 1.88 & 1.35 & 1.36 & 1.07 & 1.09 & 1.12 & 1.08 & 1.59 & 2.10 & 1.46 & 1.45 & 1.57 \\
\hline $\mathrm{P}_{2} \mathrm{O}_{5}$ & 0.46 & 0.43 & 0.44 & 0.39 & 0.39 & 0.44 & 0.45 & 0.51 & 0.34 & 0.23 & 0.24 & 0.21 & 0.21 & 0.17 \\
\hline LOI & & & 0.45 & 0.14 & 0.25 & 0.96 & 0.25 & 0.32 & 0.54 & 0.42 & 1.28 & 0.78 & 0.77 & 0.31 \\
\hline Total & 99.84 & 99.85 & 100.04 & 99.95 & 100.10 & 100.54 & 100.47 & 100.35 & 98.61 & 98.61 & 98.90 & 98.84 & 98.98 & 98.97 \\
\hline $\mathrm{Li}$ & & & & 6.4 & 6.0 & & & 5.7 & 8.8 & 15.3 & 18.7 & 12.2 & 13.1 & \\
\hline $\mathrm{Be}$ & & & & 0.98 & 1.13 & & & 3.41 & 0.88 & 1.14 & 1.37 & 0.88 & 0.88 & \\
\hline $\mathrm{Sc}$ & 26 & 25 & 26 & 28 & 30 & 21 & 22 & 26 & 24 & 7 & 5 & 16 & 16 & 20 \\
\hline $\mathrm{V}$ & 212 & 210 & 216 & 243 & 243 & 167 & 156 & 269 & 214 & 78 & 56 & 172 & 172 & 157 \\
\hline $\mathrm{Cr}$ & 255 & 260 & 220 & 545 & 539 & 340 & 362 & 237 & 191 & 11 & 8 & 49 & 68 & 48 \\
\hline Co & 38 & 40 & 37 & 43 & 47 & 40 & 40 & 32 & 30 & 5 & 3 & 22 & 20 & 18 \\
\hline $\mathrm{Ni}$ & 133 & 131 & 129 & 230 & 227 & 249 & 257 & 80 & 38 & 0 & 0 & 17 & 23 & 17 \\
\hline $\mathrm{Cu}$ & & & & 76 & 34 & & & 19 & 35 & 12 & 7 & 47 & 34 & \\
\hline $\mathrm{Zn}$ & 70 & 69 & 83 & 75 & 76 & 86 & 80 & 106 & 93 & 64 & 65 & 60 & 62 & 57 \\
\hline $\mathrm{Ga}$ & 16 & 18 & 18 & 17 & 15 & 16 & 18 & 19 & 16 & 17 & 17 & 22 & 19 & 17 \\
\hline $\mathrm{Rb}$ & 22 & 24 & 23 & 26 & 28 & 13 & 16 & 13 & 0 & 28 & 42 & 33 & 31 & 33 \\
\hline $\mathrm{Sr}$ & 626 & 629 & 614 & 558 & 554 & 538 & 550 & 846 & 591 & 492 & 540 & 480 & 478 & 456 \\
\hline $\mathrm{Y}$ & 30 & 30 & 28 & 21 & 20 & 32 & 30 & 20 & 8 & 7 & 26 & 18 & 8 & 16 \\
\hline $\mathrm{Zr}$ & 155 & 162 & 160 & 117 & 119 & 208 & 214 & 140 & 128 & 126 & 149 & 117 & 118 & 113 \\
\hline $\mathrm{Nb}$ & & & & 7.5 & 7.5 & & & 7.3 & 6.7 & 4.1 & 5.8 & 3.5 & 3.5 & \\
\hline Cs & & & & 0.60 & 0.43 & & & 0.30 & 0.44 & 0.89 & 1.53 & 0.80 & 0.80 & \\
\hline $\mathrm{Ba}$ & 238 & 259 & 287 & 329 & 314 & 265 & 281 & 361 & 288 & 525 & 657 & 454 & 461 & 480 \\
\hline $\mathrm{La}$ & & & & 12.5 & 12.7 & & & 13.9 & 12.6 & 14.4 & 19.2 & 12.0 & 12.0 & \\
\hline $\mathrm{Ce}$ & & & & 30.8 & 31.1 & & & 35.7 & 30.5 & 31.6 & 41.4 & 26.4 & 26.5 & \\
\hline $\operatorname{Pr}$ & & & & 4.6 & 4.7 & & & 5.5 & 4.7 & 4.2 & 5.4 & 3.6 & 3.6 & \\
\hline $\mathrm{Nd}$ & & & & 20.9 & 21.2 & & & 25.5 & 21.2 & 16.7 & 20.9 & 15.1 & 15.1 & \\
\hline $\mathrm{Sm}$ & & & & 5.1 & 5.2 & & & 6.2 & 5.3 & 3.5 & 4.2 & 3.4 & 3.4 & \\
\hline $\mathrm{Eu}$ & & & & 1.49 & 1.48 & & & 1.77 & 1.52 & 1.05 & 1.16 & 1.02 & 1.01 & \\
\hline $\mathrm{Gd}$ & & & & 4.2 & 4.3 & & & 4.9 & 4.5 & 2.7 & 3.2 & 2.9 & 3.0 & \\
\hline $\mathrm{Tb}$ & & & & 0.64 & 0.64 & & & 0.73 & 0.71 & 0.44 & 0.48 & 0.45 & 0.46 & \\
\hline Dy & & & & 3.6 & 3.6 & & & 4.0 & 4.0 & 2.4 & 2.7 & 2.6 & 2.6 & \\
\hline Но & & & & 0.67 & 0.68 & & & 0.73 & 0.77 & 0.49 & 0.50 & 0.52 & 0.52 & \\
\hline $\mathrm{Er}$ & & & & 1.84 & 1.88 & & & 1.95 & 2.15 & 1.39 & 1.51 & 1.45 & 1.48 & \\
\hline $\mathrm{Tm}$ & & & & 0.26 & 0.26 & & & 0.27 & 0.31 & 0.21 & 0.24 & 0.21 & 0.22 & \\
\hline $\mathrm{Yb}$ & & & & 1.66 & 1.69 & & & 1.72 & 1.92 & 1.53 & 1.56 & 1.43 & 1.41 & \\
\hline $\mathrm{Lu}$ & & & & 0.26 & 0.25 & & & 0.25 & 0.29 & 0.23 & 0.24 & 0.22 & 0.21 & \\
\hline $\mathrm{Hf}$ & & & & 2.64 & 2.62 & & & 3.16 & 3.19 & 2.62 & 4.10 & 2.85 & 2.89 & \\
\hline $\mathrm{Ta}$ & & & & 0.48 & 0.49 & & & 0.61 & 0.48 & 0.23 & 0.31 & 0.21 & 0.20 & \\
\hline $\mathrm{Tl}$ & & & & 0.04 & 0.01 & & & 0.03 & 0.05 & 0.24 & 0.27 & 0.15 & 0.15 & \\
\hline $\mathrm{Pb}$ & & & & 1.8 & 1.5 & & & 2.9 & 2.7 & 5.7 & 8.3 & 5.0 & 4.9 & \\
\hline Th & & & & 1.31 & 1.32 & & & 1.11 & 1.24 & 2.19 & 3.57 & 1.91 & 1.86 & \\
\hline $\mathrm{U}$ & & & & 0.68 & 0.69 & & & 0.48 & 0.46 & 0.95 & 1.52 & 0.93 & 0.98 & \\
\hline
\end{tabular}


Table 1 (continued)

\begin{tabular}{|c|c|c|c|c|c|c|c|c|c|c|c|c|c|c|}
\hline \multirow{2}{*}{\multicolumn{4}{|c|}{$\begin{array}{l}\text { Bakening andesites/dacites } \\
\text { 2px-andesites }\end{array}$}} & \multicolumn{8}{|c|}{ Andesitic/dacitic centers around Bakening } & \multicolumn{3}{|c|}{ Tuya rhyodacites } \\
\hline & & & & \multicolumn{3}{|c|}{ SW bakening } & \multicolumn{5}{|c|}{ Novy bakening } & \multicolumn{2}{|c|}{$\begin{array}{l}\text { Lavaya Avacha } \\
\text { Valley }\end{array}$} & \multirow{2}{*}{$\begin{array}{l}\text { Kavyche } \\
\text { Valley } \\
\text { BAK19 }\end{array}$} \\
\hline BAK40 & $0-71$ & BAK11 & BAK13 & BAK35 & BAK36 & BAK25 & BAK03 & BAK05 & BAK09 & BAK08 & BAK07 & BAK50 & BAK51 & \\
\hline 60.68 & 61.42 & 61.72 & 61.91 & 60.19 & 60.31 & 60.34 & 62.11 & 62.52 & 62.64 & 62.71 & 62.82 & 67.25 & 72.01 & 75.06 \\
\hline 0.65 & 0.67 & 0.64 & 0.63 & 0.84 & 0.56 & 0.83 & 0.60 & 0.55 & 0.57 & 0.57 & 0.56 & 0.39 & 0.19 & 0.09 \\
\hline 16.74 & 17.15 & 16.83 & 16.54 & 17.43 & 18.19 & 17.40 & 17.57 & 17.34 & 17.49 & 17.56 & 17.53 & 16.34 & 15.16 & 14.33 \\
\hline 1.39 & 1.80 & 1.26 & 1.22 & 1.93 & 1.72 & 2.00 & 1.67 & 1.81 & 1.63 & 1.67 & 1.46 & 1.04 & 0.47 & 0.24 \\
\hline 3.89 & 3.71 & 3.74 & 3.63 & 3.46 & 1.86 & 3.49 & 2.94 & 2.50 & 2.86 & 2.76 & 2.90 & 1.89 & 0.99 & 0.71 \\
\hline 0.10 & 0.11 & 0.09 & 0.09 & 0.11 & 0.09 & 0.11 & 0.11 & 0.10 & 0.11 & 0.11 & 0.10 & 0.05 & 0.15 & 0.10 \\
\hline 3.36 & 3.51 & 2.90 & 2.85 & 2.15 & 1.39 & 2.11 & 1.62 & 1.45 & 1.54 & 1.48 & 1.48 & 1.49 & 0.48 & 0.25 \\
\hline 5.95 & 6.12 & 5.63 & 5.48 & 5.41 & 4.09 & 5.33 & 5.00 & 4.71 & 4.87 & 4.86 & 4.87 & 3.93 & 1.61 & 1.29 \\
\hline 3.95 & 4.00 & 3.98 & 3.85 & 4.39 & 4.58 & 4.48 & 4.44 & 4.38 & 4.42 & 4.38 & 4.53 & 4.62 & 5.07 & 4.43 \\
\hline 1.44 & 1.44 & 1.56 & 1.59 & 1.71 & 1.85 & 1.77 & 1.87 & 1.92 & 1.90 & 1.90 & 1.90 & 1.54 & 2.35 & 3.00 \\
\hline 0.18 & 0.20 & 0.16 & 0.16 & 0.27 & 0.24 & 0.34 & 0.26 & 0.26 & 0.26 & 0.26 & 0.26 & 0.12 & 0.14 & 0.07 \\
\hline 0.61 & 0.54 & 0.46 & 0.60 & 0.87 & 0.43 & 0.46 & 0.49 & 1.07 & 0.46 & 0.64 & 0.82 & 0.23 & 0.44 & 0.86 \\
\hline 98.95 & 100.66 & 98.95 & 98.54 & 98.74 & 95.30 & 98.65 & 98.66 & 98.60 & 98.74 & 98.90 & 99.23 & 98.89 & 99.06 & 100.44 \\
\hline 13.0 & & & 14.5 & 15.8 & & 16.0 & 16.4 & 17.8 & 17.5 & & 17.4 & 12.7 & 22.6 & 27.4 \\
\hline 0.80 & & & 0.79 & 1.20 & & 1.28 & 1.12 & 1.11 & 1.14 & & 1.11 & 0.93 & 1.70 & 1.50 \\
\hline 13 & 16 & 18 & 14 & 8 & 10 & 13 & 8 & 5 & 11 & 8 & 7 & 8 & 3 & 4 \\
\hline 139 & 143 & 137 & 137 & 128 & 70 & 125 & 78 & 66 & 73 & 72 & 72 & 71 & 7 & 5 \\
\hline 72 & 67 & 52 & 53 & 13 & 12 & 13 & 14 & 9 & 15 & 8 & 10 & 22 & 11 & 14 \\
\hline 17 & 18 & 18 & 16 & 13 & 8 & 14 & 11 & 5 & 8 & 11 & 10 & 6 & 3 & 0 \\
\hline 24 & 31 & 20 & 20 & 0 & 0 & 5 & 0 & 0 & 0 & 0 & 3 & 10 & 0 & 0 \\
\hline 35 & & & 35 & 22 & & 12 & 14 & 10 & 14 & & 11 & 45 & 17 & 2 \\
\hline 59 & 62 & 55 & 53 & 67 & 54 & 73 & 65 & 61 & 65 & 65 & 63 & 42 & 58 & 34 \\
\hline 20 & 16 & 16 & 17 & 24 & 19 & 18 & 17 & 17 & 18 & 17 & 32 & 17 & 24 & 15 \\
\hline 38 & 23 & 35 & 33 & 34 & 37 & 35 & 44 & 41 & 34 & 18 & 49 & 34 & 47 & 68 \\
\hline 471 & 465 & 482 & 446 & 525 & 518 & 589 & 506 & 487 & 506 & 495 & 498 & 655 & 324 & 213 \\
\hline 27 & 2 & 9 & 30 & 0 & 18 & 14 & 25 & 12 & 20 & 5 & 35 & 2 & 11 & 12 \\
\hline 117 & 123 & 112 & 112 & 137 & 127 & 138 & 129 & 137 & 134 & 136 & 130 & 87 & 125 & 68 \\
\hline 2.8 & & & 2.8 & 4.8 & & 5.4 & 4.3 & 4.4 & 4.3 & & 4.3 & 1.9 & 6.4 & 5.5 \\
\hline 0.76 & & & 0.93 & 1.18 & & 1.25 & 1.16 & 1.13 & 1.14 & & 1.09 & 0.29 & 1.44 & 2.15 \\
\hline 449 & 465 & 483 & 503 & 528 & 572 & 551 & 585 & 616 & 599 & 602 & 602 & 563 & 787 & 859 \\
\hline 11.4 & & & 12.0 & 14.9 & & 17.1 & 15.1 & 15.8 & 15.3 & & 15.4 & 10.9 & 14.5 & 14.5 \\
\hline 24.6 & & & 25.6 & 34.1 & & 38.4 & 32.7 & 34.0 & 33.0 & & 33.0 & 22.6 & 31.7 & 29.1 \\
\hline 3.4 & & & 3.4 & 4.7 & & 5.3 & 4.4 & 4.5 & 4.4 & & 4.4 & 2.9 & 4.3 & 3.8 \\
\hline 13.7 & & & 13.6 & 19.5 & & 21.9 & 17.6 & 18.3 & 18.0 & & 17.9 & 11.4 & 16.0 & 13.0 \\
\hline 3.0 & & & 3.0 & 4.4 & & 4.9 & 3.7 & 3.8 & 3.8 & & 3.8 & 2.3 & 3.1 & 2.4 \\
\hline 0.93 & & & 0.89 & 1.31 & & 1.33 & 1.12 & 1.12 & 1.10 & & 1.10 & 0.63 & 0.77 & 0.50 \\
\hline 2.6 & & & 2.6 & 3.7 & & 3.9 & 3.1 & 3.2 & 3.0 & & 3.0 & 1.8 & 2.2 & 1.7 \\
\hline 0.40 & & & 0.38 & 0.57 & & 0.58 & 0.48 & 0.50 & 0.48 & & 0.47 & 0.26 & 0.35 & 0.27 \\
\hline 2.3 & & & 2.3 & 3.1 & & 3.2 & 2.8 & 2.7 & 2.7 & & 2.8 & 1.4 & 2.0 & 1.5 \\
\hline 0.47 & & & 0.46 & 0.61 & & 0.62 & 0.53 & 0.55 & 0.55 & & 0.55 & 0.26 & 0.41 & 0.28 \\
\hline 1.32 & & & 1.30 & 1.71 & & 1.72 & 1.60 & 1.63 & 1.59 & & 1.53 & 0.72 & 1.24 & 0.84 \\
\hline 0.19 & & & 0.18 & 0.26 & & 0.25 & 0.25 & 0.24 & 0.24 & & 0.25 & 0.11 & 0.20 & 0.13 \\
\hline 1.29 & & & 1.27 & 1.70 & & 1.71 & 1.62 & 1.72 & 1.64 & & 1.60 & 0.65 & 1.41 & 0.89 \\
\hline 0.20 & & & 0.19 & 0.25 & & 0.25 & 0.25 & 0.26 & 0.25 & & 0.24 & 0.10 & 0.23 & 0.13 \\
\hline 2.91 & & & 2.95 & 3.47 & & 3.59 & 3.25 & 3.56 & 3.39 & & 3.35 & 2.80 & 2.85 & 2.06 \\
\hline 0.18 & & & 0.20 & 0.26 & & 0.27 & 0.24 & 0.26 & 0.25 & & 0.23 & 0.17 & 0.46 & 0.50 \\
\hline 0.16 & & & 0.19 & 0.17 & & 0.18 & 0.21 & 0.22 & 0.18 & & 0.21 & 0.16 & 0.30 & 0.44 \\
\hline 4.9 & & & 5.7 & 6.3 & & 6.6 & 6.5 & 6.7 & 6.4 & & 6.7 & 6.7 & 11.4 & 8.5 \\
\hline 2.04 & & & 1.98 & 2.60 & & 2.77 & 2.67 & 2.87 & 2.80 & & 2.64 & 2.13 & 2.08 & 3.28 \\
\hline 0.88 & & & 1.06 & 1.15 & & 1.23 & 1.29 & 1.36 & 1.33 & & 1.33 & 0.98 & 1.23 & 2.05 \\
\hline
\end{tabular}




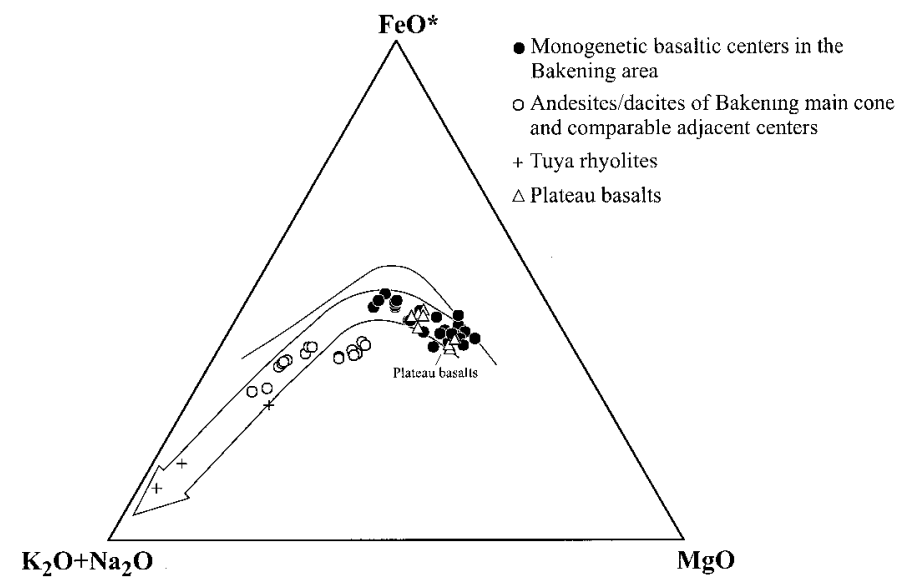

Fig. 2. AFM diagram, showing the lava compositions of the different Quaternary rock types in the Bakening area. All studied rocks fall into the calc-alkaline field (boundary after Irvine and Baragar, 1971). The plateau basalts and Late Quaternary monogenetic basaltic centers follow the same trend. The dacitic and rhyodacitic samples are separated from the basaltic rocks and show strong Fe and Mg depletion.

The Bakening stratovolcano is composed of andesites and dacites with $\mathrm{SiO}_{2}$ ranging from 60 to $66 \%$. Small isolated mostly dacitic centers are mineralogically and geochemically similar. A gap in composition exists between the basaltic and dacitic suites from 56 to $60 \% \mathrm{SiO}_{2}$. Concentrations of $\mathrm{FeO}, \mathrm{CaO}, \mathrm{MnO}$, $\mathrm{MgO}$ and $\mathrm{TiO}_{2}$ are lower and $\mathrm{Na}_{2} \mathrm{O}$ and $\mathrm{K}_{2} \mathrm{O}$ higher compared to the basaltic rocks (Fig. 3). A series of younger amphibole-free flows covers the Bakening stratovolcano, These are the least-evolved rocks of the stratocone and form different trends compared to the other andesites and dacites.

\subsection{Trace elements and isotopes}

Variations for certain trace elements with $\mathrm{MgO}$ are shown in Fig. 4. Compatible trace elements such as $\mathrm{Sc}, \mathrm{Co}, \mathrm{V}, \mathrm{Cr}$ and $\mathrm{Ni}$ have high abundances in the basaltic rocks and show a strong negative correlation with $\mathrm{MgO}$ and the Mg\#. Sample BAK24 contains $500 \mathrm{ppm} \mathrm{Cr}$ and $170 \mathrm{ppm} \mathrm{Ni}$ at a $\mathrm{Mg \#}$ of 65 and represents the most primitive near-primary magma. In comparison, dacitic rocks are more depleted in compatible elements. Incompatible trace elements such as $\mathrm{Ba}, \mathrm{Pb}, \mathrm{Zr}, \mathrm{Nb}$ and the $\mathrm{La} / \mathrm{Yb}$ ratio increase slightly with decreasing $\mathrm{MgO}$ in the basaltic and stronger in the dacitic rocks. Sr contents increase in the basalts but decrease only slightly in the dacitic rocks. This behavior is comparable with that of $\mathrm{Al}_{2} \mathrm{O}_{3}$ (Figs. 3 and 4).

Again the two basaltic samples O35 and BAK64 are enriched in some trace elements $(\mathrm{Sr}, \mathrm{Zr}, \mathrm{Nb}$ and $\mathrm{La} / \mathrm{Y}$ ) and show similarity to the plateau basalts, which are also enriched in all HFSE, $\mathrm{Sr}$ and the LREE relative to the normal Bakening basalts.

Cumulate inclusions were found to consist of $\sim 20 \%$ amphibole, $\sim 40 \%$ plagioclase and $\sim 40 \%$ interstitial glass. From one cumulate, enclosed in an amphibole-bearing dacite amphibole and plagioclase were mechanically separated and measured by ICPMS for trace elements (Table 2). The amphibole of the cumulate is enriched in the middle to heavy REE relative to its dacitic host (Table 3 ). The highest enrichment $(3 \times)$ is found for Dy. REE concentrations in plagioclase are negligibly low. These measurements are in agreement with the reported mineral/melt distribution coefficients in andesitic and dacitic systems (Fujimaki et al., 1984; Dunn and Sen, 1994; Sisson, 1994; LaTourrette et al., 1995).

The overall trace element pattern for Late Quaternary rocks is generally similar and typical for island arc rocks (Fig. 5). However, in detail, there are some unusual features (see below). Fluid mobile trace elements are strongly enriched and HFS elements are relatively depleted. The enrichment decreases with decreasing element incompatibility. $\mathrm{Ba}, \mathrm{Rb}, \mathrm{U}$, 

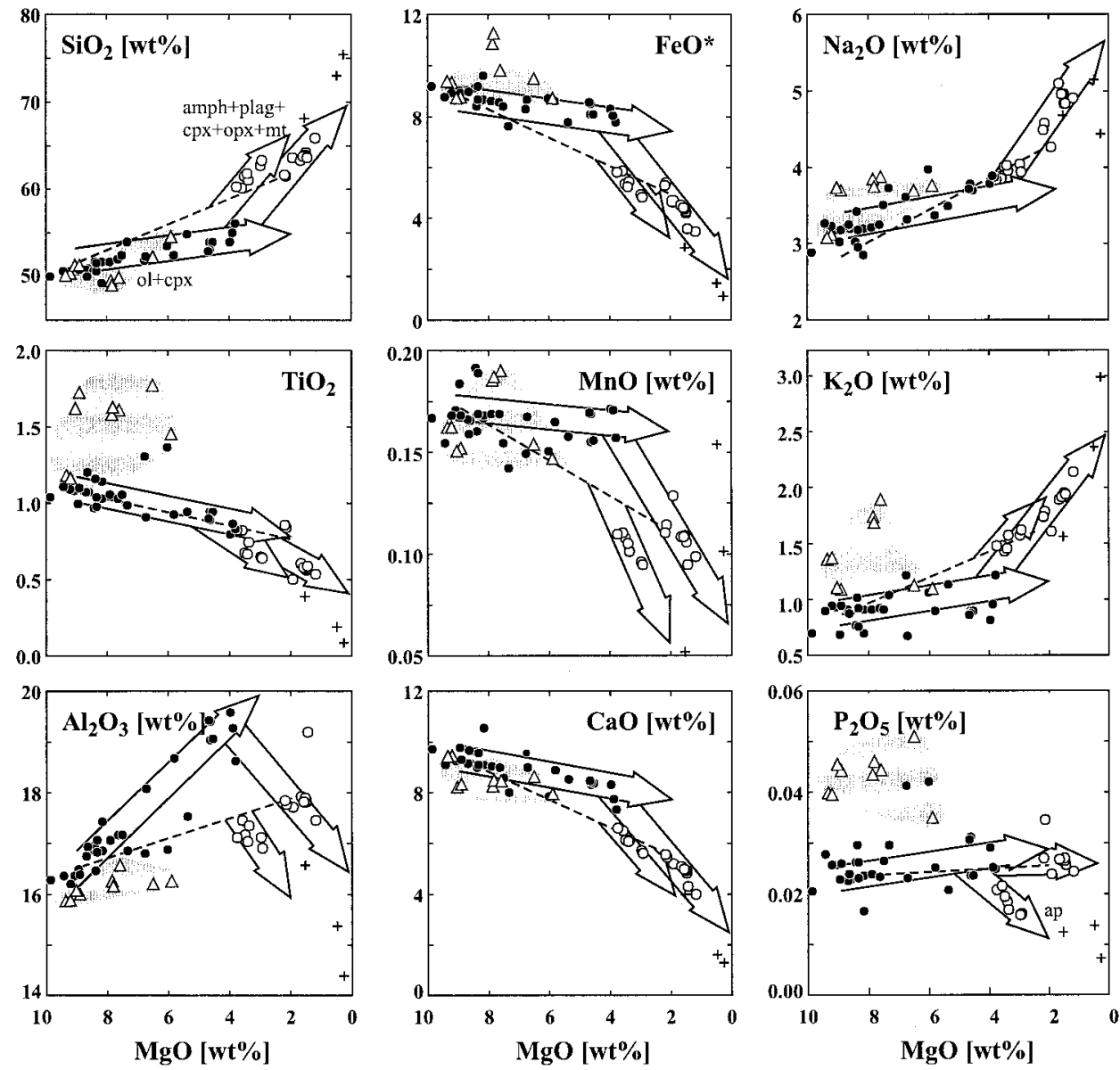

Fig. 3. Major elements variations versus MgO. Symbols as in Fig. 2. The different trends in the Upper Quaternary basaltic and dacitic rocks are caused by a variable mineral fractionation, which is emphasized by the arrows. The Plateau basalts are displaced in most diagrams from these trends. Some dacitic rocks are affected by mixing, which is outlined by the dashed line.

Th and $\mathrm{K}$ have more than 10 times higher concentrations compared to NMORB. Dacitic rocks show an even stronger enrichment of the most incompatible over less incompatible elements than the basaltic rocks. $\mathrm{Nb}$ and Ta have low concentrations especially in the basaltic rocks similar to NMORB. HREE are more strongly depleted in the dacites while the LREE are more enriched than in the mafic monogeneitic centers. This results in a cross-over of the REE pattern for rocks of the Bakening area (Fig. 6). The plateau basalts mostly overlap with the Bakening basalts, except in their less-pronounced HFSE depletion.

$\mathrm{The} \mathrm{Sr}, \mathrm{Nd}$ and $\mathrm{Pb}$ isotopic compositions are similar for all rocks in the Bakening area and only slightly displaced from MORB (Table 4). Their variations are inside the field for other Quaternary volcanics of Kamchatka (Churikova et al., 2000).

\section{Discussion}

A model for the genesis of arc magmatism at Bakening volcano needs to concentrate on the systematics of trace elements and their differences between the andesitc to dacitic lavas from the Bakening stratovolcano, the monogenetic basaltic centers in 

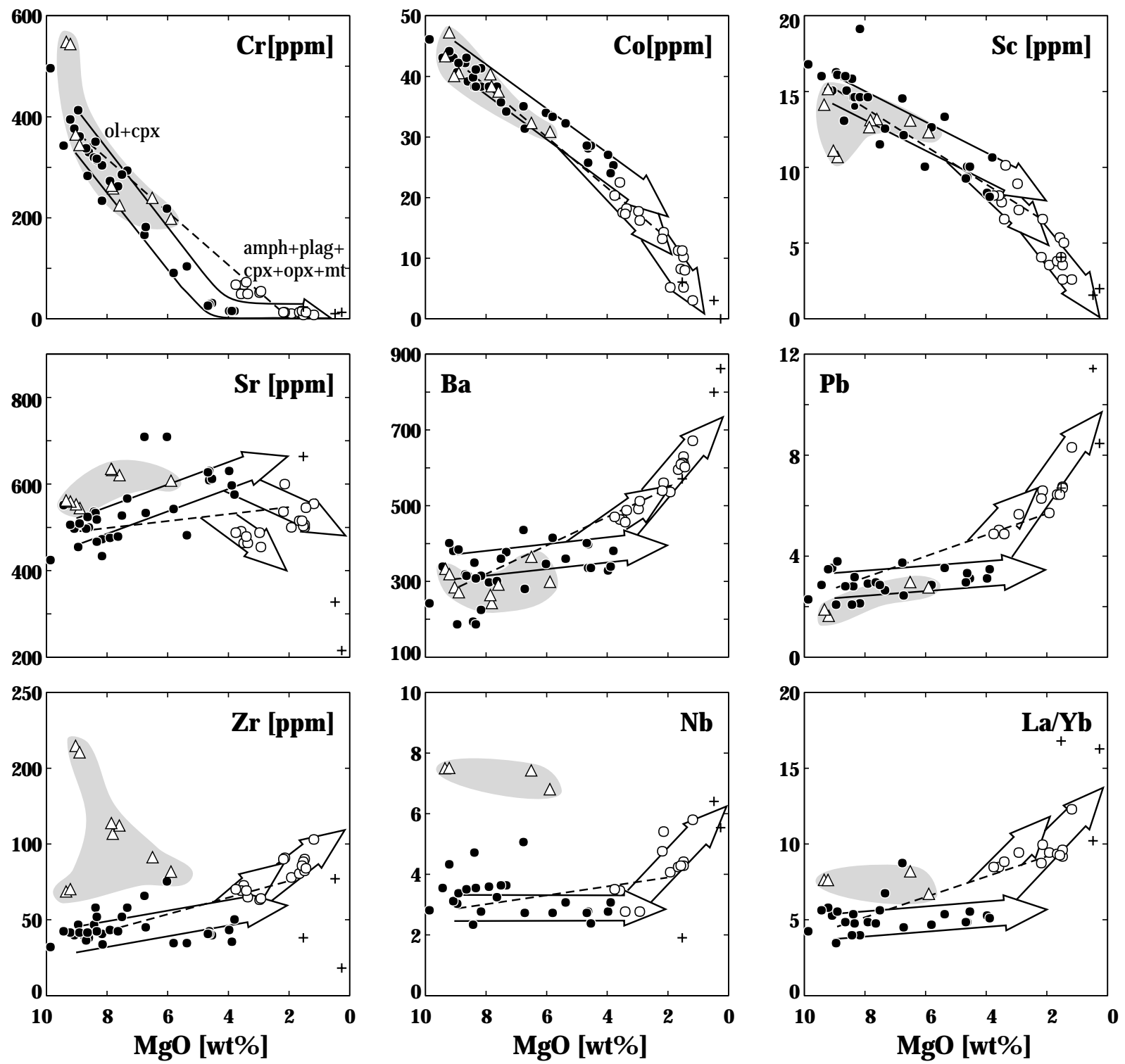

Fig. 4. Trace elements variations versus MgO. Symbols as in Fig. 2. The different fractionating phases cause the different trends in the basaltic and dacitic rocks. Fractionation trends are indicated by arrows, the mixing between basaltic and dacitic melts by a dashed line. The Plateau basalts have distinctive composition for some trace elements compared to the Upper Quaternary basaltic rocks.

the surrounding areas and the older, Pleistocene Plateau basalts. The main feature and differences are summarized below:

(1) All lavas from the Bakening area are characterized by relatively high $\mathrm{Sr} / \mathrm{Y}$ ratios compared to lavas from the Eastern Volcanic front and the Central Kamchatka depression. This parameter that has been used to identify adakitic slab melts (Defant and Drummond, 1990). However, true adakites have $\mathrm{Sr} / \mathrm{Y}$ up to 300 because of much higher Sr contents than any of the Bakening lavas.

(2) LREE over HREE enrichment (i.e. high and variable $\mathrm{Ce} / \mathrm{Yb}$ ratios) are observed, but this steepening of the REE pattern does not extend to the HREE. 
Table 2

REE-data of mineral separates of amphibole and plagioclase from the cumulate BAK63 (WR) determined by ICPMS (all values in ppm; b.d.l. = below detection limit)

\begin{tabular}{lccll}
\hline & Amphibole & Plagioclase & Amph/WR & Plag/WR \\
\hline $\mathrm{La}$ & 1.8 & 2.5 & 0.1 & 0.2 \\
$\mathrm{Ce}$ & 7.8 & 3.9 & 0.3 & 0.1 \\
$\mathrm{Pr}$ & 1.8 & 0.4 & 0.5 & 0.1 \\
$\mathrm{Nd}$ & 12.4 & 1.4 & 0.8 & 0.1 \\
$\mathrm{Sm}$ & 5.0 & 0.1 & $\mathbf{1 . 7}$ & 0.0 \\
$\mathrm{Eu}$ & 1.6 & 0,4 & $\mathbf{2 . 0}$ & 0.5 \\
$\mathrm{Gd}$ & 5.4 & 0.1 & $\mathbf{2 . 3}$ & 0.0 \\
$\mathrm{~Tb}$ & 0.9 & b.d.1. & $\mathbf{2 . 7}$ & \\
$\mathrm{Dy}$ & 5.5 & 0.0 & $\mathbf{3 . 0}$ & 0.0 \\
$\mathrm{Ho}$ & 1.1 & b.d.1. & $\mathbf{2 . 9}$ & \\
$\mathrm{Er}$ & 2.9 & b.d.1. & $\mathbf{2 . 6}$ & \\
$\mathrm{Tm}$ & 0.4 & b.d.1. & $\mathbf{2 . 4}$ & \\
$\mathrm{Yb}$ & 2.3 & b.d.1. & $\mathbf{2 . 0}$ & \\
$\mathrm{Lu}$ & 0.3 & b.d.1. & $\mathbf{1 . 8}$ & \\
$\mathrm{Nb}$ & 1.1 & b.d.1. & 0.3 & \\
$\mathrm{Zr}$ & 43 & 1.0 & 0.4 & 0.0 \\
$\mathrm{~Pb}$ & 2.9 & 1.3 & 0.5 & 0.2 \\
$\mathrm{Sr}$ & 265 & 1020 & 0.6 & $\mathbf{2 . 4}$ \\
$\mathrm{Ba}$ & 80 & 100 & 0.1 & 0.1 \\
\hline
\end{tabular}

This is seen from the overall REE enrichment, which is not accompanied by steepening of the HREE (Figs. 6 and 7). Taking all Bakening data together, there is a range of $\mathrm{Ce} / \mathrm{Yb}$ ratios for a given $\mathrm{Yb}$ content. Closer inspection, however, shows that within each rock

Table 3

Last square calculation for the REE pattern of the dacitic rocks, using $D_{\text {mineral/melt }}$ values of Fujimaki (1986) and Fujimaki et al. (1984). The best fit was found for fractionation of $20 \% \mathrm{amph}$, $12 \%$ plag, $7 \% \mathrm{cpx}$ and $0.6 \%$ apatite. All concentrations in ppm

\begin{tabular}{lrrc}
\hline & Parent (BAK-42) & Daughter (BAK-03) & Calc. daughter \\
\hline $\mathrm{La}$ & 11.63 & 15.10 & 14.94 \\
$\mathrm{Ce}$ & 28.27 & 32.71 & 33.15 \\
$\mathrm{Pr}$ & 4.26 & 4.40 & 4.54 \\
$\mathrm{Nd}$ & 19.26 & 17.63 & 18.63 \\
$\mathrm{Sm}$ & 4.62 & 3.72 & 3.66 \\
$\mathrm{Eu}$ & 1.36 & 1.12 & 1.10 \\
$\mathrm{Gd}$ & 3.90 & 3.07 & 3.02 \\
$\mathrm{~Tb}$ & 0.60 & 0.48 & 0.47 \\
$\mathrm{Dy}$ & 3.35 & 2.75 & 2.66 \\
$\mathrm{Ho}$ & 0.63 & 0.53 & 0.53 \\
$\mathrm{Er}$ & 1.83 & 1.60 & 1.65 \\
$\mathrm{Tm}$ & 0.26 & 0.25 & 0.24 \\
$\mathrm{Yb}$ & 1.72 & 1.62 & 1.63 \\
$\mathrm{Lu}$ & 0.25 & 0.25 & 0.25 \\
\hline
\end{tabular}

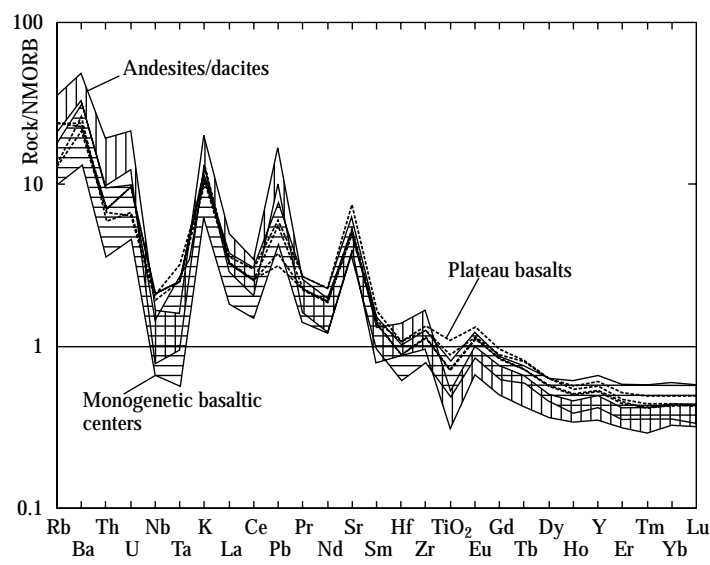

Fig. 5. MORB-normalized trace element patterns of volcanic rocks in the Bakening area. For clarity, the monogenetic basalts and andesite/dacite rocks of Holocene to Upper Pleistocene age are shown as compositional fields, which are based on 28 and 12 analyses, respectively. Three analyses of plateau basalts are shown for comparison. The normalization values for NMORB and the element order are those from Hofmann (1988). The similar pattern of all three groups is remarkable. In detail however, dacitic rocks display higher concentrations in the very incompatible elements but lower concentrations in HREE than the basaltic rocks. Plateau basalts differ by the less pronounced depletion in HFS elements from the other rocks and a steeper HREE pattern.

series as defined above, there is in fact a range in $\mathrm{Yb}$ with a horizontal trend (no change) in $\mathrm{Ce} / \mathrm{Yb}$ : the overall variation is composed of several stacked subhorizontal trends. This observation clearly argues against the role of garnet in the source of Bakening stratovolcano lavas and the mafic monogenetic centers. Garnet in the residue would produce constant $\mathrm{Yb}$ at variable $\mathrm{Ce} / \mathrm{Yb}$ ratios and fractionated HREE within each rock series, which is not observed. From this, we argue that slab melting is not a viable process in the origin of the Bakening lavas, that a high $\mathrm{Sr} / \mathrm{Y}$ ratio may not be an universal indication of slab melts (Defant et al., 1991) and that other processes, such as amphibole-dominated fractionation need to be considered as an alternative process.

(3) The plateau lavas are different, they do show a tendency of higher with larger HREE fractionation, similar to the rocks from the northern end of the Central Kamchatka depression (Shiveluch, Kharzinsky, Zarechny). These lavas therefore may have an adakitic signature (Yogodinsky et al., submitted).

We now evaluate a fractional crystallization 


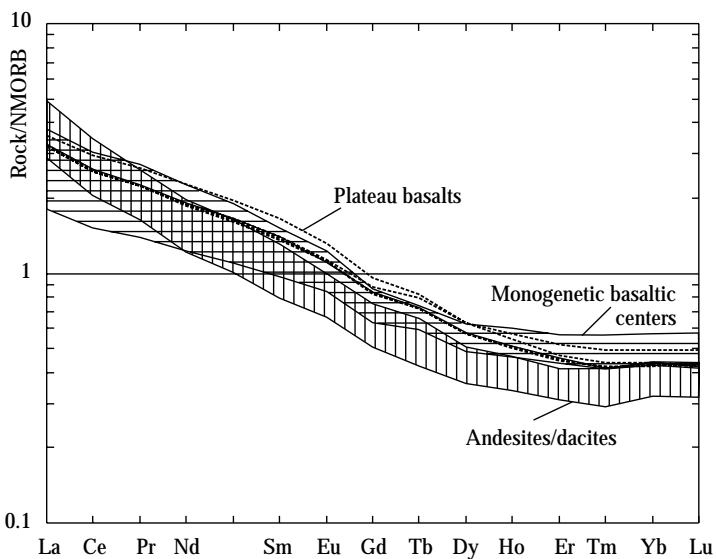

Fig. 6. REE variation in rocks from the Bakening area normalized to NMORB (Hofmann, 1988). All suites are depleted in HREE and enriched in LREE concentrations compared to NMORB. Plateau basalts have steeper pattern than the younger basaltic rocks. The dacitic rocks are HREE depleted and LREE enriched, resulting in the cross-over of the pattern. However, the HREE distribution is rather flat and unfractionated.

model for the compositional variation within the mafic suite and between the mafic rocks and the evolved andesite and dacite lavas from the Bakening stratovolcano.

We will, however, come back to the discussion of a possible role of adakites in the genesis of Bakening Plateau basalt lavas.

\subsection{Fractional crystallization}

Some of the differences in major and trace elements are consistent with changes caused by mineral fractionation. Here we first examine the fractionation history of the mafic rocks of the Bakening area.

\subsubsection{Basaltic monogenetic centers}

Major element variations in this suite are mainly

Table 4

$\mathrm{Sr}-$, Nd-, and $\mathrm{Pb}$-isotope composition of Quaternary volcanics in the Bakening area

\begin{tabular}{|c|c|c|c|c|c|}
\hline & ${ }^{87} \mathrm{Sr} /{ }^{86} \mathrm{Sr}$ & ${ }^{143} \mathrm{Nd} /{ }^{144} \mathrm{Nd}$ & ${ }^{206} \mathrm{~Pb} /{ }^{204} \mathrm{~Pb}$ & ${ }^{207} \mathrm{~Pb} /{ }^{204} \mathrm{~Pb}$ & ${ }^{208} \mathrm{~Pb} /{ }^{204} \mathrm{~Pb}$ \\
\hline \multicolumn{6}{|c|}{ Upper Quaternary basaltic rocks from monogenetic centers } \\
\hline BAK04 & 0.70311 & 0.51307 & 18.347 & 15.492 & 38.015 \\
\hline BAK18 & 0.70317 & 0.51307 & & & \\
\hline BAK17 & 0.70315 & 0.51307 & 18.298 & 15.493 & 37.998 \\
\hline BAK24 & 0.70317 & 0.51306 & 18.345 & 15.501 & 38.062 \\
\hline BAK30 & 0.70327 & 0.51304 & & & \\
\hline BAK31 & 0.70329 & 0.51303 & 18.334 & 15.482 & 38.032 \\
\hline $\mathrm{O} 26$ & 0.70322 & 0.51307 & & & \\
\hline \multicolumn{6}{|c|}{ Lower Quaternary plateau basalt } \\
\hline BAK06 & 0.70337 & 0.51303 & & & \\
\hline \multicolumn{6}{|c|}{ Upper Quaternary dacitic rocks } \\
\hline BAK09 & 0.70329 & 0.51302 & 18.279 & 15.468 & 37.921 \\
\hline BAK10 & 0.70327 & 0.51304 & & & \\
\hline BAK39 & 0.70332 & 0.51306 & 18.281 & 15.472 & 37.924 \\
\hline \multicolumn{6}{|c|}{ Late Pleistocene tuja rhyodacite } \\
\hline BAK19 & 0.70322 & 0.51308 & & & \\
\hline
\end{tabular}




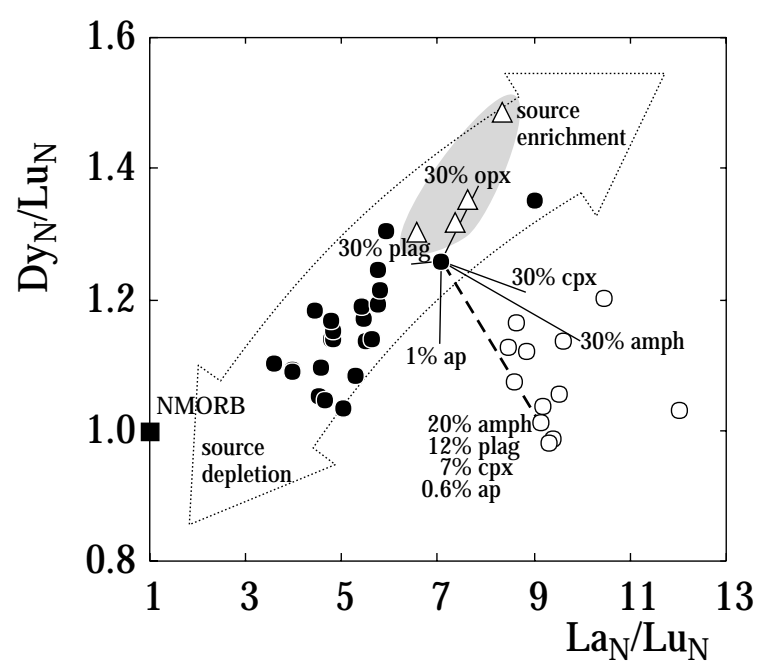

Fig. 7. NMORB-normalized element ratios (Hofmann, 1988) of $\mathrm{La} / \mathrm{Lu}$ versus $\mathrm{Dy} / \mathrm{Lu}$ to distinguish between fractionation and melting processes, which influence the REE pattern. All samples are displaced to considerable higher $\mathrm{La} / \mathrm{Lu}$ ratios compared to MORB, which can be explained by slab fluid enrichment. The positive trend in the basaltic samples, including plateau basalts is caused by mixing between depleted and enriched mantle components. The cause of this enrichment remains unclear (see text for discussion). The dacitic samples are strongly influenced by amphibole fractionation. Vectors for the different phases and the calculated fractionation assemblage are shown. These vectors are only applicable to dacitic samples. The fractionation of cpx, ol and plag has a negligible effect in basaltic rocks, because of the much lower mineral/melt partition coefficients.

controlled by fractionation of olivine and clinopyroxene, which strongly deplete the magma in $\mathrm{MgO}, \mathrm{CaO}, \mathrm{FeO}$ and compatible trace elements $(\mathrm{Cr}$, $\mathrm{Co}, \mathrm{Ni}, \mathrm{Sc}, \mathrm{V})$. Linear trends indicate that the ratio between the fractionating minerals was more or less constant, regardless of the observed range in phenocryst abundances. Significant plagioclase and amphibole fractionation can be excluded for the majority of the samples from $\mathrm{MgO}$ versus $\mathrm{Al}_{2} \mathrm{O}_{3}$ and $\mathrm{Sr}$ trends (Figs. 3 and 4). Only at $\mathrm{MgO}<5 \%$ does minor plagioclase fractionation occur, which is evident from decreasing $\mathrm{Al}_{2} \mathrm{O}_{3}$. The slightly alkaline basalts O35 and BAK64, which lack plagioclase phenocrysts fall off this fractionation trend. They probably formed from a distinct parental magma, which was enriched in $\mathrm{Ti}, \mathrm{Na}$ and $\mathrm{P}$, similar to that of the plateau basalts. The linear trend of decreasing $\mathrm{TiO}_{2}$ with decreasing $\mathrm{MgO}$ in the basaltic samples is problematic, because all fractionating phases, except magnetite, have $D_{\text {mineral } / \text { melt }}<1$ for Ti. There is no indication for early amphibole crystallization in the basaltic magmas. The observed $\mathrm{Ti}$ depletion in the basaltic rocks can be attained by only $\sim 1 \%$ magnetite fractionation. However, (titano-) magnetite as phenocryst was detected in only three of 31 samples. Two additional samples from these same lava flows did not contain magnetite, despite similar Ti-concentrations. This indicates that magnetite crystallization in the basaltic magmas occurred just before or during eruption and thus had no influence on $\mathrm{TiO}_{2}$ depletion. Clinopyroxene could also be more Ti-rich than predicted by most of the experimentally determined distribution coefficients in basaltic systems. Typical hour-glass zonation structures in fact indicate that the clinopyroxenes have relatively high Ti-contents. Mixing between mafic and Ti-poor evolved magmas is not feasible because the intermediate lavas have the highest $\mathrm{Al}$ contents and do not fall on a mixing line. We thus favor the fractionation hypothesis for the reduction in $\mathrm{Ti}$, but this would involve fractionation of a non-modal assemblage.

\subsubsection{Andesite and dacite suite of Bakening stratovolcano}

Mineralogy and major element trends of the dacitic suite are consistent with a process of fractionation of amphibole, plagioclase, ortho- and clinopyroxene, magnetite and apatite from an evolved member of the basaltic suite (Fig. 3). This assemblage has a relatively low $\mathrm{MgO}$ and high $\mathrm{SiO}_{2}$ content compared to the fractionating minerals in the basalts, which cause different element trends versus $\mathrm{MgO}$ and $\mathrm{SiO}_{2}$ for the two suites. The gap in $\mathrm{SiO}_{2}$ between the basaltic and dacitic rocks can be explained by extensive plagioclase and amphibole (both minerals are enriched in $\mathrm{SiO}_{2}$ and depleted in $\mathrm{MgO}$ compared to the melt) fractionation in the magma chamber before eruption. This is suggested by the fact that no gap exists for $\mathrm{MgO}$, which is not much affected by plagioclase and amphibole fractionation. Plagioclase fractionation is also evident from the decrease in $\mathrm{Al}_{2} \mathrm{O}_{3}$ and $\mathrm{Sr}$.

The decrease in Ti continues in the dacitic rocks. However magnetite is abundant in all dacitic rocks (1\%) and easily explains this observation. Up to $0.5 \%$ apatite fractionation must have occurred to 

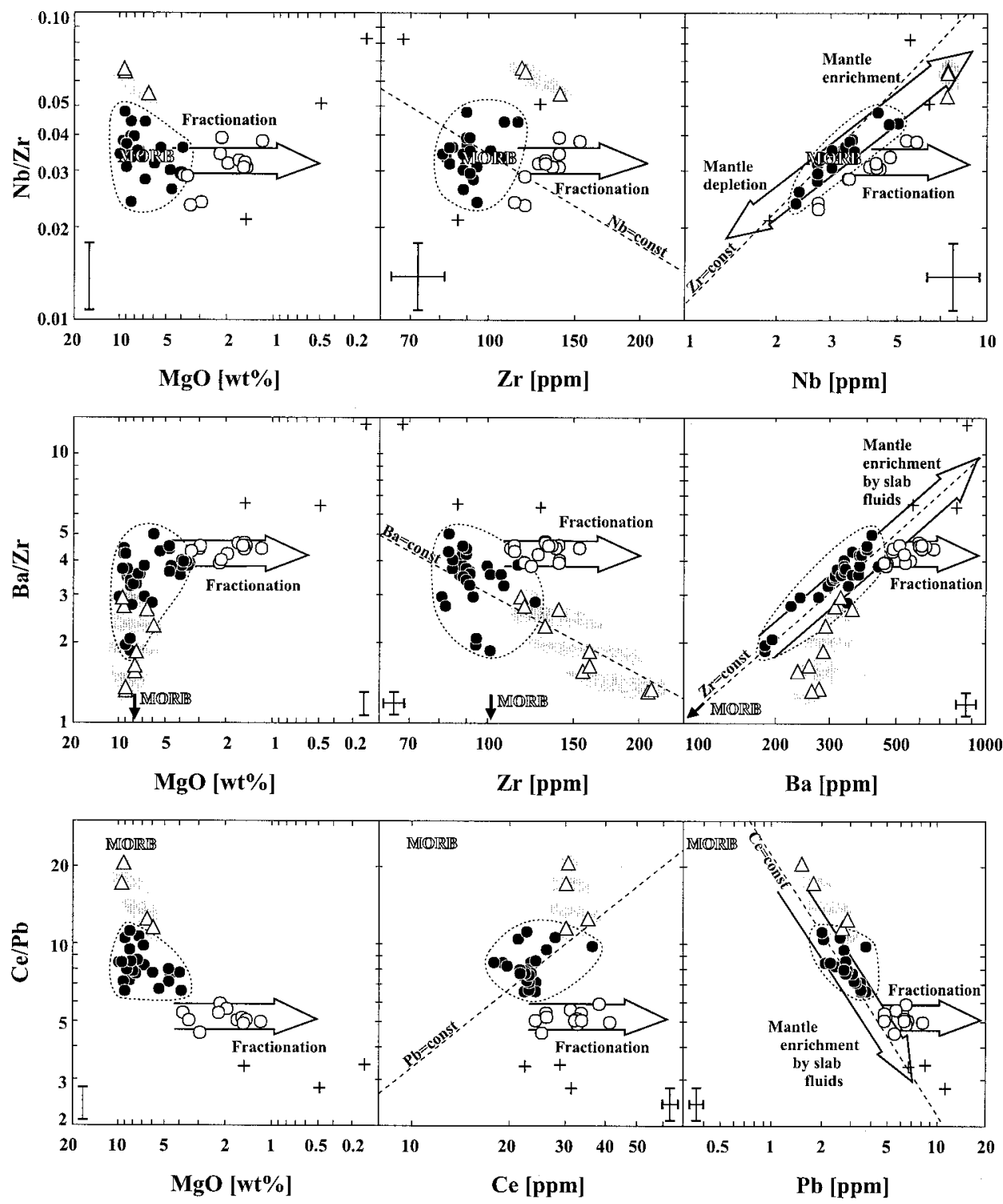

Fig. 8. Incompatible trace element ratios, used to distinguish between source depletion and enrichment processes. $\mathrm{MgO}$ and incompatible elements are used as fractionation index. Fractionation or different degrees of mantle melting should result in horizontal trends. Any correlations therefore have to be caused by variable source compositions. See text for discussion.

produce the decrease of phosphorus in the dacitic rocks (Fig. 3). From the relative enrichment of highly incompatible elements $(\mathrm{Ba}, \mathrm{Rb}, \mathrm{U}, \mathrm{Th})$, the degree of fractionation can be estimated. If the most primitive basalt BAK24 is chosen as parent, between 50 and $80 \%$ fractionation must have occurred to produce the dacitic rocks.
The youngest cpx-opx-andesite flows form a subgroup in the stratovolcano suite. They are more mafic (Fig. 3) and their trends are distinct from that of other andesites and dacites. Major and trace element data suggest that the parental magma may have formed by mixing of a basaltic magma into the dacitic magma chamber of Bakening volcano (dashed 


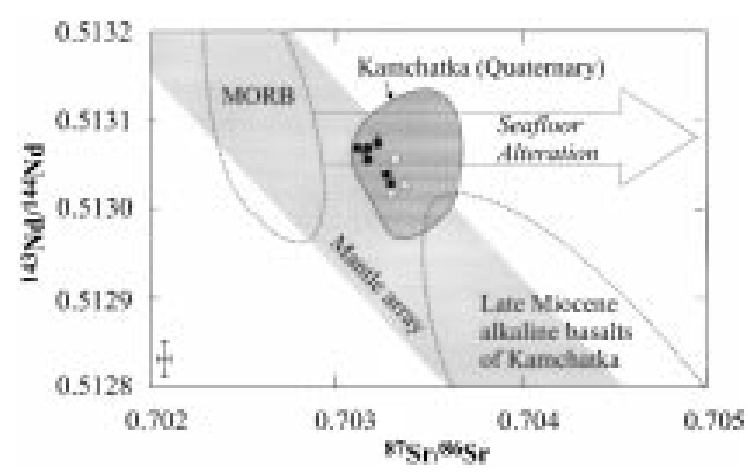

Fig. 9. Sr and Nd isotopes of rocks from the Bakening area. The MORB field (Macdougall and Lugmair, 1986; Ito and Stern, 1986; White et al., 1987) and the field for Miocene alkali basalts (Volynets et al., 1997) are added for comparison. The values for Bakening are rather primitive and show no significant internal trends. The slight displacement from the mantle array to a more radiogenic Sr-isotopic composition maybe caused by slab fluids derived from the altered oceanic crust. Such seafloor alteration change the Sr-isotopic ratios but has no influence on Nd. Same symbols as in Fig. 2.

lines in Figs. 3 and 4). Hybrid magmas (70\% dacitic and $30 \%$ basalt) must have homogenized and further fractionated before eruption because the analyzed samples fall away from a hypothetical mixing line. Occasionally plagioclase occurs with sieve structures and olivines rimmed with fine-grained aggregates of orthopyroxene indicating disequilibrium conditions on the microscopic scale and support the mixing hypothesis.

\subsection{REE-pattern}

Increasing REE contents and increasing LREE/ HREE ratios from basaltic to dacitic rocks cannot be caused mainly by fractionation of olivine, pyroxene and plagioclase. The decrease in MREE and HREE contents in the dacitic compared to the basaltic rocks needs a different explanation (Fig. 7). Amphibole has $D_{\text {mineral } / \text { melt }}>1$ for the MREE and HREE in andesites and dacites. Apatite can also have a significant influence on the REE pattern despite its small amount in the fractionation assemblage because it has very high $D_{\text {mineral/melt }}$ for all REE.

A least squares calculation relating a primitive basaltic sample (BAK24) and a dacite (BAK03) by fractionation of $20 \%$ amphibole, $12 \%$ plagioclase, $7 \%$ clinopyroxene and $0.6 \%$ apatite yields a good approximation of the observed REE (Table 3, Fig. 7). $D_{\text {mineral/melt }}$ values for calc-alkaline andesites (Fujimaki et al., 1984; Fujimaki, 1986) were used in the calculation. Additional evidence for an amphiboledominated fractionation in the evolved rocks comes from amphibole-rich cumulates, occasionally occurring in the older Bakening dacites. The high analyzed REE-contents of amphibole from such cumulate (Table 2) emphasize the role of amphibole for the HREE-depletion in the dacitic rocks.

With a dominance of amphibole in the fractionating assemblage between basalts and dacites it is thus possible to explain the variable LREE/HREE ratios at low MREE/HREE abundances. It may also explain the relatively high $\mathrm{Sr} / \mathrm{Y}$ ratios and therefore this parameter may not be a universal indicator of slab melts.

\subsection{Source composition}

Modeling of the observed HREE contents of basaltic rocks by melting a NMORB source gives high melting rates of about $30 \%$. This is higher than the 10-25\% melting inferred by Plank and Langmuir (1993) for typical arc volcanism. If the NMORB source is previously depleted by $10 \%$ melt extraction we get more reasonable melting degrees of $15-20 \%$. Residual phases may also influence on the REE pattern. At low melting degrees garnet, clinopyroxene or amphibole in the residuum can produce relatively HREE-depleted melts. At $15 \%$ melting degree of an ultra-depleted mantle, both phases are completely consumed in the melt and will have no influence on the REE-pattern. Garnet as residual phase can be stable at low melting degrees. However its influence must be limited, because it should produce a large range in the LREE/HREE ratios in basalts and fractionate the HREE, which is not observed in our samples.

Mantle depletion is expressed in the $\mathrm{Nb} / \mathrm{Zr}$ ratio as well because $\mathrm{Nb}$ is stronger depleted compared to $\mathrm{Zr}$ after mantle melting. Both elements are not influenced by fluid enrichment (Ayers and Eggler, 1995; Brenan et al., 1995b). The $\mathrm{Nb} / \mathrm{Zr}$ ratio is also not influenced by fractional crystallization (Fig. 8 and Table 3 ). $\mathrm{Nb}$ / $\mathrm{Zr}$ against $\mathrm{Nb}$ shows a positive trend in the basaltic and dacitic rocks. This trend is partly compromised by the relatively large analytical error for $\mathrm{Nb}$. However, 
all plateau basalt and some Late Quaternary basaltic rocks are clearly displaced to higher $\mathrm{Zr}$ and $\mathrm{Nb}$ concentrations and higher $\mathrm{Nb} / \mathrm{Zr}$ ratios. The $\mathrm{Zr}$ enrichment is even more pronounced in $\mathrm{Zr}$ versus $\mathrm{Ba} / \mathrm{Zr}$, where at a nearly constant $\mathrm{Ba}$ concentration $\mathrm{Zr}$ is strongly enriched. This higher HFSE concentration can result from a more primitive, less-depleted mantle source. The $\mathrm{Nb} / \mathrm{Zr}$ ratios of the Bakening rocks range from enriched to depleted compared to NMORB. We believe that this variation represents mixing between variably depleted mantle sources.

\subsection{Fluid enrichment in the source}

Ratios of incompatible elements can be used to get information about the magma source composition. The dacitic suite and the rhyodacites, however, have to be evaluated with caution because even fractionation of minor phases like biotite, apatite and zircon, occurring in such evolved rocks in low abundances can influence the fluid-mobile elements. Also substantial amphibole fractionation can influence incompatible element ratios.

With this precautions in mind, ratios of fluid and melt mobile (K, Rb, Ba, U, Th) over fluid-immobile but melt-mobile ( $\mathrm{Zr}, \mathrm{Hf}, \mathrm{Nb}, \mathrm{Ta})$ trace elements are a powerful tool to study source enrichment by slab derived fluids. For example the $\mathrm{Ba} / \mathrm{Zr}$ ratio in the basaltic suite is variable from 1.8 to 4.9 (Fig. 8). Dacitic samples have 3.8-4.6 $\mathrm{Ba} / \mathrm{Zr}$ and are inside the basaltic range. Plateau basalts are lowest in $\mathrm{Ba} / \mathrm{Zr}$, from 1.3 to 2.6, caused by the high $\mathrm{Zr}$ concentrations in more mafic samples.

The $\mathrm{Ba} / \mathrm{Zr}$ ratio in basaltic samples is independent of their degree of differentiation (i.e. $\mathrm{MgO}$ content). Therefore, fractionation did not affect this ratio. It is also unlikely, that variable degrees of partial melting have affected $\mathrm{Ba} / \mathrm{Zr}$ because of high melting degrees. We conclude that variable $\mathrm{Ba} / \mathrm{Zr}$ ratios reflect slightly distinct mantle sources, probably affected by variable degrees of slab-fluid enrichment.

Miller et al. (1994) used the $\mathrm{Ce} / \mathrm{Pb}$ ratio as tracer for the amount of slab fluids involved in the source. Such fluids are highly enriched in $\mathrm{Pb}$ compared to the REE (Brenan et al., 1995a) and therefore variations in fluid enrichment, e.g. variable $\mathrm{Ba} / \mathrm{Zr}$ ratios should anticorrelate with $\mathrm{Ce} / \mathrm{Pb}$. The $\mathrm{Ce} / \mathrm{Pb}$ ratios in the basaltic suite are variably low from 6.6 to 11.2. In comparison, the $\mathrm{Ce} / \mathrm{Pb}$ ratios in dacitic samples reach from 4.5 to 5.8 , i.e. they are lower and less variable than in the basaltic rocks. The nearly constant $\mathrm{Ce} / \mathrm{Pb}$ in the andesitic to dacitic rocks with varaiable $\mathrm{MgO}$ suggests that the $\mathrm{Ce} / \mathrm{Pb}$ ratio is not changed by fractionation up to dacitic compositions and that the low ratios are a characteristic of the parent and its source. A variable fluid-induced $\mathrm{Pb}$ enrichment of the source is shown by positive $\mathrm{Pb}$ spike in the trace element patterns (Fig. 5) and the negative trend between $\mathrm{Ce} / \mathrm{Pb}$ and $\mathrm{Pb}$ in the basaltic rocks. The $\mathrm{Ba} /$ $\mathrm{Zr}$ ratios are slightly anti-correlated with $\mathrm{Ce} / \mathrm{Pb}$, as expected. This suggests that these ratios are largely controlled by variable fluid component in the source.

\subsection{Isotopic constraints}

$\mathrm{Sr}-, \mathrm{Pb}-$ and $\mathrm{Nd}$-isotope ratios are close to the MORB field (Fig. 9). In $\mathrm{Sr}-\mathrm{Nd}$ space, the data are shifted slightly to a more radiogenic $\mathrm{Sr}$ isotopic composition, compared to MORB, but still within the mantle array. Kamchatka volcanic rocks from all other analyzed volcanoes (Churikova et al., in revision) define a field slightly displaced from the mantle array towards more radiogenic $\mathrm{Sr}$ isotopes (Fig. 9). This small shift was interpreted as the result of a Sr-rich slab fluid mixed into the strongly depleted mantle wedge (Kepezhinskas et al., 1997; Churikova et al., 2000; Dorendorf et al., 2000). The amount of sediment in the source for Kamchatka arc rocks was estimated on the basis of $\mathrm{Pb}-, \mathrm{Sr}-, \mathrm{Nd}-$ and Be-isotopes to be less than 1\% (Kersting and Arculus, 1995; Bailey, 1996).

The dacitic suite is slightly more radiogenic in $\mathrm{Sr}$. However, there is no trend with $\mathrm{SiO}_{2}$ or $\mathrm{Mg \#}$ and the most-evolved rhyodacite BAK19 has an isotopic composition identical within error to the basaltic rocks. This argues against a significant role of assimilation of old crust. The Sr-isotope ratio from one analyzed plateau basalt is more radiogenic, but still within the field for typical Late Quaternary rocks.

\subsection{Source of the platean basalts}

The number of studied plateau basalts analyzed here is limited and only preliminary interpretations can be given. All plateau basalts have LILE concentrations comparable or even slightly higher than Late 
Quaternary Bakening rocks. Most remarkable is the relative enrichment in Ti, Na, K, P, Sr, HFSE, LREE and depletion in $\mathrm{Ca}$ and $\mathrm{Al}$. This results in low LILE/ HFSE and high LREE/HREE ratios. High $\mathrm{Ce} / \mathrm{Pb}$ ratios between 11 and 20 and low $\mathrm{Ba} / \mathrm{Zr}$ ratios are caused by high $\mathrm{Zr}$ and $\mathrm{Ce}$ concentrations (Fig. 8). The $\mathrm{Zr}$ contents decrease with an increase in $\mathrm{SiO}_{2}$ or decrease in $\mathrm{MgO}$ (Fig. 4) and some elements $\left(\mathrm{K}_{2} \mathrm{O}\right.$ 1.1-1.9\%; Sr: 540-850 ppm) are strongly scattered, excluding, that these rocks are derived from one source. The HREE are similarly depleted as in the Late Quaternary basalts in absolute abundances, but a distinctive feature are the more strongly fractionated HREE (higher Dy $(n) / \mathrm{Lu}(n)$, Fig. 7).

Fluids alone cannot explain this pattern and the differences with the other rocks at Bakening. One possible option is a small contribution of a slab melt in the source. Slab melts are dacitic, they are enriched in LILE and LREE, depleted in HREE and have high Sr/Y (Defant and Drummond, 1990). Such compositions were in fact observed by Kepezhinskas and Defant (1996) in glasses from xenoliths sampled in plateau basalts $\mathrm{N}$ of Bakening. Clinopyroxenes from such metasomatized xenoliths were described to be enriched in $\mathrm{Al}, \mathrm{Ti}, \mathrm{Na}, \mathrm{Sr}, \mathrm{Nb}, \mathrm{Zr}$ and $\mathrm{REE}$ compared to unmetasomatized xenoliths. The metasomatizing component is characterized by a high $\mathrm{La} / \mathrm{Yb}, \mathrm{Zr} / \mathrm{Hf}, \mathrm{Sr} / \mathrm{Sm}, \mathrm{Nb} / \mathrm{La}$ and low Ti/Eu and $\mathrm{Y} / \mathrm{Er}$.

If indeed these features are interpreted to derive from a slab melt component, several problems arise. The subducted slab below Bakening volcano is of Lower Cretaceous age and far too old and cold to melt (Peacock et al., 1994; Peacock, 1996). There is no evidence for a tear or a window in the slab, therefore no slab-side effect is possible (Yogodinsky et al., in revision).

As an alternative, the plateau basalts could have been produced by fluid-induced melting of a mantle source that consists of a depleted and a slightly enriched (OIB-type) component. HFSE elements are not carried by the fluid and their enrichment therefore could be caused by a mixture between depleted mantle wedge and an enriched component in the source. A similar two-component mixing model was developed by Volynets et al. (1997) for Miocene basalts from the Valaginsky Range in Eastern Kamchatka and in some rocks in the back arc region of the SR by Churikova et al. (in revision). Both argue from Sr- and Nd-isotope variations for mixing of an OIB-type mantle into the arc magma source in these areas. However, our isotopic compositions of Bakening plateau basalts are not more enriched than the younger monogenetic basalts. Two of the Late Quaternary basaltic rocks (O35, BAK64) show a tendency to a plateau basalt trace element pattern and would suggest that such a source mix may have survived and was involved in magma genesis until recently.

Geochemical data on the plateau basalts are limited and we cannot make a clear case for either of the two possible models. Therefore, the supposed slabsignature (Kepezhinskas and Defant, 1996) for the source of the Plateau basalts remains enigmatic and warrants further study.

\section{Conclusions}

Major elements show a close relationship between the bimodal Late Quaternary basaltic and dacitic suites in the Bakening area. The basaltic rocks start by fractionation olivine and clinopyroxene, but plagioclase becomes more abundant as the magma evolves. Fractionation in dacitic rocks is controlled by amphibole, plagioclase, clinopyroxene, orthopyroxene and minor magnetite and apatite.

Basaltic samples show a range of variably depleted sources, overprinted by different degrees of fluid enrichment. Dacitic rocks have a similar trace element pattern as that of the basaltic rocks (Fig. 5). Slight differences in LILE and the remarkable HREE depletion can be produced by amphibole fractionation. The $\mathrm{Ba} / \mathrm{Zr}$ and $\mathrm{Ce} / \mathrm{Pb}$ ratios of the dacitic suite indicate derivation from a source, which was similar or even more enriched in fluid mobile elements like the most enriched source of the basaltic suite. Plateau basalts were either derived from a mixture of depleted and enriched sources that were overprinted by the slab fluid or the higher $\mathrm{Sr} / \mathrm{Y}$ rations and HREE fractionation indicated a slab component in the source.

\section{Acknowledgements}

This work was supported by DFG-project Wo362/ 15-1 +2 and a grant by the Volkswagen-Foundation to G.W. Further support came from the Russian grant 
No. 94-3129 (T.C.), an INTAS-project granted to Clive Oppenheimer (Bristol), and by grant No. 9805-04103 from DFG-RFBR Programm. Critical reviews by P. Kepezhinskas and P. Kyle were helpful in improving an earlier version of the manuscript. We are very grateful to Dr I. Melekestsev and O. Dirksen for help and collaboration during the field work.

\section{References}

Ayers, J.C., Eggler, D.H., 1995. Partitioning of elements between silicate melt and $\mathrm{H} 2 \mathrm{O}-\mathrm{NaCl}$ fluids at 1.5 and $2.0 \mathrm{GPa}$ pressure; implications for mantle metasomatism. Geochimica et Cosmochimica Acta 59 (20), 4237-4246.

Bailey, J.C., 1996. Role of subducted sediments in the genesis of Kurile-Kamchatka island arc basalts; $\mathrm{Sr}$ isotopic and elemental evidence. Geochemical Journal 30 (5), 289-321.

Brenan, J.M., Shaw, H.F., Ryerson, F.J., 1995a. Experimntal evidence for the origin of lead enrichment in convergent-margin magmas. Nature (London) 378 (6552), 54-56.

Brenan, J.M., Shaw, H.F., Ryerson, F.J., Phinney, D.L., 1995b. Mineral-aqueous fluid partitioning of trace elements at 900 degrees $\mathrm{C}$ and $2.0 \mathrm{GPa}$; constraints on the trace element chemistry of mantle and deep crustal fluids. Geochimica et Cosmochimica Acta 59 (16), 3331-3350.

Churikova, T., Dorendorf, F., Wörner, G., 2000. Sources and fluids in the mantle wedge below Kamchatka, evidence from acrossarc geochemical variation (submitted for publication).

Defant, M.J., Drummond, M.S., 1990. Derivation of some modern arc magmas by melting of young subducted lithosphere. Nature (London) 347 (6294), 662-665.

Defant, M.J., Richerson, P.M., de-Boer, J.Z., Stewart, R.H., Maury, R.C., Bellon, H., Drummond, M.S., Feigenson, M.D., Jackson, T.E., 1991. Dacite Genesis via both slab melting and differentiation: petrogenesis of La Yeguada volcanic complex, Panama. Journal of Petrology 32 (6), 1101-1142.

Dirksen, O.V., Melekestsev, I.V., 1998. Monogenetic volcanism at the vicinity of Bakening volcano (Kamchatka, Russia). In: International Seismic Volcanic Workshop on Kamchatkan-Aleutian Subdaction Processes, IVGG FED RAS. PetropavlovskKamchatsky, Russia, pp. 35-36.

Dorendorf, F., Wiechert, U., Wörner, G., 2000. Hydrated sub-arc mantle: a source for Kluchevskoy volcano/Kamchatka. Earth and Planetary Science Letters 175, 69-86.

Dunn, T., Sen, C., 1994. Mineral/ matrix partition coefficients for orthopyroxene, plagioclase, and olivine in basaltic to andesitic systems; a combined analytical and experimental study. Geochimica et Cosmochimica Acta 58 (2), 717-733.

Fujimaki, H., 1986. Partition coefficients of Hf, Zr, and REE between zircon, apatite, and liquid. Contributions to Mineralogy and Petrology 94 (1), 42-45.

Fujimaki, H., Tatsumoto, M., Aoki, K.I., 1984. Partitioning coefficients of $\mathrm{Hf}, \mathrm{Zr}$, and REE between phenocrysts and groundmasses. In: Boynton, W.V., Schubert, G. (Eds.), Proceedings of the Fourteenth Lunar and Planetary Science Conference;
Part 2. Journal of Geophysical Research. B. 89, Suppl., Washington, DC, pp. 662-672.

Hochstaedter, A.G., Kepezhinskas, P., Defant, M., Drummond, M., Koloskov, A., 1996. Insights into the volcanic arc mantle wedge from magnesian lavas from the Kamchatka Arc. Journal of Geophysical Research, B 101, 697-712.

Hofmann, A.W., 1988. Chemical differentiation of the Earth; the relationship between mantle, continental crust, and oceanic crust. In: Welin, E. (Ed.). Isotope geochemistry; the Crafoord symposium. Earth and Planetary Science Letters, Elsevier, Amsterdam, pp. 297-314.

Irvine, T.N., Baragar, W.R., 1971. A guide to the chemical classification of the common volcanic rocks. Canadian Journal of Earth Sciences 8 (5), 523-547.

Ito, E., Stern, R.J., 1986. Oxygen- and strontium-isotopic investigations of subduction zone volcanism; the case of the Volcano Arc and the Marianas island arc. Earth and Planetary Science Letters $76(3 / 4), 312-320$.

Kepezhinskas, P., Defant, M.J., Drummond, M.S., 1996. Progressive enrichment of island arc mantle by melt-peridotite interaction inferred from Kamchatka xenoliths. Geochimica et Cosmochimica Acta 60 (7), 1217-1229.

Kepezhinskas, P., McDermott, F., Defant, M.J., Hochstaedter, A.G., Drummond, M.S., Hawkesworth, C.J., Koloskov, A.V., Maury, R.C., Bellon, H., 1997. Trace element and $\mathrm{Sr}-\mathrm{Nd}-\mathrm{Pb}$ isotopic constraints on a three-component model of Kamchatka Arc petrogenesis. Geochimica et Cosmochimica Acta 61 (3), 577600.

Kersting, A.B., Arculus, R.J., 1995. Pb isotope composition of Klyuchevskoy Volcano, Kamchatka and North Pacific sediments; implications for magma genesis and crustal recycling in the Kamchatkan arc. Earth and Planetary Science Letters 136 (3/4), 133-148.

Koloskov, A.V., Volynets, O.N., Ponomarev, G.P., Fedorov, P.I., 1997. Ultramafic xenoliths in island-arc volcanic rocks of various geochemical type. Petrology 5 (5), 431-447.

LaTourrette, T., Hervig, R.L., Holloway, J.R., 1995. Trace element partitioning between amphibole, phlogopite, and basanite melt. Earth and Planetary Science Letters 135 (1-4), 13-30.

Macdougall, J.D., Lugmair, G.W., 1986. Sr and $\mathrm{Nd}$ isotopes in basalts from the East Pacific Rise; significance for mantle heterogeneity. Earth and Planetary Science Letters 77 (3/4), 273-284.

Melekestsev, I.V., Dirksen, O.V., Girina, O.A., 1988. A giant explosive-landslide cirque and a detrical avalanche on Bakening volcano, Kamchatka. Vulkanologija and Seismologija 3, 12-24 (in Russian, with English abstract).

Miller, D.M., Goldstein, S.L., Langmuir, C.H., 1994. Cerium/lead and lead isotope ratios in arc magmas and the enrichment of lead in the continents. Nature (London) 368 (6471), 514-520.

Peacock, S.M., 1996. Thermal and petrologic structure of subduction zones. In: Bebout, G.E., Scholl, D.W., Kirby, S.H., Platt, J.P. (Eds.), Subduction top to bottom. Geophysical Monograph. vol. 96. pp. 119-133.

Peacock, S.M., Rushmer, T., Thompson, A.B., 1994. Partial melting of subducting oceanic crust. Earth and Planetary Science Letters $121,227-244$. 
Piip, B.I., 1947. Field-geological studies in South Kamchatka. Trudy Kamchatskoi Vulkanologicheskoi Stantsii 3 (in Russian).

Plank, T., Langmuir, C.H., 1993. Tracing trace elements from sediment input to volcanic output at subduction zones. Nature (London) 362 (6422), 739-743.

Sisson, T.W., 1994. Hornblende-melt trace-element partitioning measured by ion microprobe. In: Foley, S.F., van-der-LaanSieger, R. (Eds.), Trace-element partitioning with application to magmatic processes. Chemical Geology 117 (1-4), 331-344.

Svjatlowsky, A.E., 1956. History of recent volcanism in the area of the Bakening volcano. Trudy Laboratorii Vulkanologii 12 (in Russian).

Todt, W., Cliff, R.A., Hanser, A., Hofmann, A.W., 1984. 202Pb205Pb spike for $\mathrm{Pb}$ isotope analysis. Terra Cognita 4, 209.

Turner, S.P., McDermott, F., Hawkesworth, C.J., Kepezhinskas, P., 1998. A U-series study of lavas from Kamchatka and the Aleutians; constraints on source composition and melting processes. Contributions to Mineralogy and Petrology 133 (3), 217-234.

Volynets, O.N., 1994. Geochemical types, petrology, and genesis of Late Cenozoic volcanic rocks from the Kurile-Kamchatka island-arc system. International Geology Review 36 (4), $373-$ 405.

Volynets, O.N., Karpenko, S.F., Kay, R.W., Gorring, M., 1997. Isotopic composition of Late Neogene $\mathrm{K}-\mathrm{Na}$ alkaline basalts of Eastern Kamchatka: indikators of the heterogeneity of the mantle magma sources. Geochemistry International 35, 884896.

White, W.M., Hofmann, A.W., Puchelt, H., 1987. Isotope geochemistry of Pacific mid-ocean ridge basalt. Journal of Geophysical Research 92 (6), 4881-4893.

Zjurupa, A.I., 1978. Erosion of the Bakening Caldera; a conceptual analysis of its genesis. Bulletin Volcanologicheskoi Stancii (in Russian). 